\title{
Numerical Simulations of in Vitro Nanoparticle Toxicity - the Case of Poly(amido amine) Dendrimers.
}

\author{
Marcus Maher \\ Marcus.Maher@mydit.ie \\ Pratap Naha \\ Technological University Dublin, pratap.naha@tudublin.ie \\ Sourav Prasanna Mukherjee \\ sourav.mukherjee@tudublin.ie
}

See next page for additional authors

Follow this and additional works at: https://arrow.tudublin.ie/nanolart

Part of the Biochemistry Commons, Biophysics Commons, Molecular Biology Commons, Other

Biochemistry, Biophysics, and Structural Biology Commons, Other Pharmacology, Toxicology and

Environmental Health Commons, Pharmacology Commons, Structural Biology Commons, and the

Toxicology Commons

\section{Recommended Citation}

Maher, M. A. et al. (2014) Numerical simulations of in vitro nanoparticle toxicity - the case of Poly(amido amine) dendrimers, Toxicology In Vitro, 28, pp. 1449-1460. doi:10.1016/j.tiv.2014.07.014

This Article is brought to you for free and open access by the NanoLab at ARROW@TU Dublin. It has been accepted for inclusion in Articles by an authorized administrator of ARROW@TU Dublin. For more information, please contact arrow.admin@tudublin.ie, aisling.coyne@tudublin.ie,gerard.connolly@tudublin.ie. Funder: DIT 
Authors

Marcus Maher, Pratap Naha, Sourav Prasanna Mukherjee, and Hugh Byrne

This article is available at ARROW@TU Dublin: https://arrow.tudublin.ie/nanolart/54 


\section{$\underline{\text { Numerical simulations of in vitro nanoparticle toxicity - the case of }}$}

\section{$\underline{\text { Poly(amido amine) dendrimers. }}$}

Marcus A. Maher ${ }^{*}$, Pratap C. Naha ${ }^{\dagger}$, Sourav Prassana Mukerjee ${ }^{\S}$, Hugh J. Byrne

Focas Research Institute, Dublin Institute of Technology, Kevin Street, Dublin 8, Ireland.

$\dagger$ Now at: Department of Radiology, Perelman School of Medicine, University of Pennsylvania, Philadelphia, Pennsylvania 19104, USA

$\S$ Now at: Health Canada, Mechanistic Studies Division, Ottawa, Ontario, Canada

*Corresponding Author:

Marcus A. Maher,

Focas Research Institute,

Dublin Institute of Technology,

Kevin Street, Dublin 8, Ireland.

E mail address: marcus.maher@mydit.ie 


\begin{abstract}
$\underline{\text { Abstract }}$
A phenomenological rate equation model is constructed to numerically simulate nanoparticle uptake and subsequent cellular response. Polyamidoamine dendrimers (generations 4-6) are modelled and the temporal evolution of the intracellular cascade of; increased levels of reactive oxygen species, intracellular antioxidant species, caspase activation, mitochondrial membrane potential decay, tumour necrosis factor and interleukin generation is simulated, based on experimental observations.
\end{abstract}

The dose and generation dependence of several of these response factors are seen to well represent experimental observations at a range of time points. The model indicates that variations between responses of different cell-lines, including murine macrophages, human keratinocytes and colon cells, can be simulated and understood in terms of different intracellular antioxidant levels, and, within a given cell-line, varying responses of different cytotoxicity assays can be understood in terms of their sensitivities to different intracellular cascade events.

The model serves as a tool to interpolate and visualise the range of dose and temporal dependences and elucidate the mechanisms underlying the in vitro cytotoxic response to nanoparticle exposure and describes the interaction in terms of independent nanoparticle properties and cellular parameters, based on reaction rates. Such an approach could be a valid alternative to that of effective concentrations for classification of nanotoxicity and may lay the foundation for future quantitative structure activity relationships and predictive nanotoxicity models. 


\section{$\underline{\text { Introduction }}$}

Research involving nanoparticles has seen a huge increase in recent years. This is undoubtedly due to the novel properties these particles possess and their potential uses in a variety of fields including: medicine, electronics, engineering, cosmetics, food, textiles, packaging and many more. ${ }^{1}$

Medical applications is a sector where nanomaterials have shown great potential and have already been used in several areas including: in vivo and in vitro diagnostic tools, biocompatible materials for implants, nutraceuticals, cancer therapy and, in particular, drug delivery. ${ }^{2-8}$ In terms of drug delivery, this interest is due to properties such as the ability to cross biological barriers easier than some more traditional delivery vehicles and the potential to escape from intracellular compartments such as lysosomes and endosomes. ${ }^{9}$ A full list of properties and potential uses for nanoparticles in drug delivery is somewhat outside the scope of this paper and has been reviewed elsewhere (De Jong et al., 2008). ${ }^{10}$ While the avenue of new medical applications does look promising, it has been found that some nanoparticles, when exposed to mammalian cells, elicit a toxic response. ${ }^{11}$

In vitro studies indicate this toxicity to be the result of oxidative stress, manifest as increased Reactive Oxygen Species (ROS) production shortly after endocytosis, ${ }^{12}$ with subsequent trafficking of the nanoparticle seen to occur through endosomes and lysosomes. ${ }^{13,14}$ The oxidative stress leads to a release of inflammatory factors ${ }^{15,16,17,18,19,20, \text { reviewed by } 21}$ and potential activation of apoptotic pathways..$^{22,23,24,25}$ It is also important to consider processes such as endosomal rupture (endosomolysis), ${ }^{9,14}$ which can enhance the toxic response as released nanoparticles have been shown to localise in and subsequently damage organelles such as the 
mitochondria. ${ }^{15,16}$ If medical applications are to remain a viable option for nanomaterials, then it will be essential to explore and better define the mechanisms involved in the toxic response.

However, in the majority of current studies, in vitro toxicity is quantified using the effective concentration for $50 \%$ loss of viability $\left(\mathrm{EC}_{50}\right)$ endpoint as an indicator of overall toxic effect. The $\mathrm{EC}_{50}$, is the result of a complex cascade of events which occur between the initial exposure and cell death; it gives no indication of the mechanisms, kinetics or efficacy of the interim processes. Additionally, the measured $\mathrm{EC}_{50}$ is dependent on the assay employed and the responses of different cell lines to the same exposure conditions have been shown to vary significantly. ${ }^{16}$ Coupled with the broad range of nanoparticle compositions, structures, sizes and possible surface functionalisations, the result is a vast array of studies from which it is difficult to derive clear systematic trends.

A more rationalised approach to nanotoxicity classification is becoming increasingly important because, as more and more nanoparticles are made available, testing via a case-by-case approach will not be sufficient. ${ }^{26,27}$ If nano-toxicology, and by extension nanomedicine, is to advance, the focus should be on:

(i) Identification of the particle based properties which induce the cellular response,

(ii) The cellular parameters which result in variations in that response, and

(iii) The variability introduced by the use of different assays.

By doing this, it may be easier to elucidate processes and events which are common to a large set of nanoparticles. Initial screening methods should be conducted in vitro, as there is a drive for a reduction in the use of animal models for evaluating toxicity, due to regulatory developments in both the EU and US (EU Directive-2010/63/EU and US Public Law 106-545, 2010, 106th 
Congress) $)^{28,29}$ generally based on the 3 R's of Russell and Burch $^{30}$ to replace, reduce and refine the use of animals used for scientific purposes. Therefore, there is currently much promotion of the development of in vitro models which can accurately infer in vivo results. One strategy to help meet these requirements, recently endorsed by the $\mathrm{OECD},{ }^{31}$ is the analysis of Adverse Outcome Pathways (AOPs), by which a sequential chain of causally linked events at different levels of biological organization that lead to an adverse health or ecotoxicological effect is identified. The aim of the development of AOP is to i) guide the development of (in vitro) Test Guidelines, ii) provide a basis for the design of Integrated Approaches to Testing and Assessment (IATA), and iii) guide the development of molecular profilers for the QSAR toolbox. A QSAR ${ }^{32}$ (Quantitative Structure Activity Relationship) can be used to identify and model traits which are common to entire sets of nanoparticles and hopefully elucidate how these properties/traits impact the overall toxicity. By advancing the knowledge of these models, it is hoped that, eventually, it will be viable to predict the full toxic profile of a cell which has taken up a nanoparticle. ${ }^{33}$

In the field of nano-toxicology (in vitro), the endpoint is usually the median $\mathrm{EC}_{50}$ of a colorometric assay, but the choice of both assay and cell-line is large and little consideration has been given to the different modes of action of the assay within the cell. Many studies have explored the mechanisms underlying the toxic response, but little attention has been devoted to quantifiable comparison between different particles or cell lines, and relationship between endpoint and nanoparticle properties. The domain of nanoparticles is vast, but the paucity of systematic studies renders it difficult to establish domains of applicability of any structureactivity paradigm, and therefore to define unambiguous algorithms or to validate based on statistical goodness-of-fit, robustness and predictability. 
In the drive to develop a better understanding of the structural dependence of toxicity and mechanisms, studies using a homologous series of nanoparticles with systematically varied physico-chemical properties can play a vital role. Systematic variations of cellular uptake and mechanisms of response, such as oxidative stress and inflammatory responses can be compared to the systematic changes in the physico-chemical properties of the nanoparticle. A better quantitative understanding of the mechanisms of response allows a better insight into the function of the cytotoxicity assays, and how the endpoints vary systematically with nanoparticle properties, but also with cell line, ultimately laying the foundation for the development of quantitatively predictive models for cellular response. ${ }^{27}$

Polyamidoamine (PAMAM) dendrimer nanoparticles are a homologous series of nanoparticles of well defined physico-chemical properties which are systematically variable and elicit systematically variable cellular responses. PAMAM dendrimers are branched in conformation and consist of three main parts, (i) the initiator core, (ii) the interior branches and (iii) the exposed branch termini. ${ }^{34}$ Each set of these branches is called a Generation $(G)$ and the generation determines the number of surface amino groups according to the formula:

$$
\mathrm{N}_{\mathrm{amg}}=\mathrm{N}_{\mathrm{BP}(\mathrm{G} 0)} \cdot 2^{\mathrm{G}}
$$

Equation (1)

where $N_{a m g}$ is the number of surface amino groups, $N_{B P(G 0)}$ is the number of initial branching points at generation zero $(G O)$ and $G$ denotes the generation number. Thus, the diameter and number of surface amino-groups increases systematically with increasing generation.

This well defined branched system allows for the variation of parameters such as: Terminal modification via addition of cationic, anionic or neutral molecules, zeta potential and in particular generation, which will govern: number of amino groups and therefore effective surface 
charge, diameter and overall particle size. Ultimately this system lets us examine how these characteristics impact the cell-nanoparticle interaction and therefore may shed light on the processes involved in toxicity. Previous studies of exposure of aquatic species ${ }^{35}$ and in vitro mammalian cell cultures ${ }^{16,23}$ have demonstrated that the polymeric dendrimer series with systematically varied structures elicits toxic responses which are well correlated with the variations in physico-chemical properties.

Uptake of these PAMAM dendrimers occurs via endocytosis, where the nanoparticle is enveloped in cellular membrane and transported into the cell. ${ }^{23,36,37}$ The toxic response has been shown to derive from an increased production of intracellular Reactive Oxygen Species (ROS) after endocytosis. ${ }^{17}$ The ROS production is counteracted by cellular anti-oxidants, one example of these being Glutathione (GSH), a thiol based tri-peptide. ${ }^{38}$ After this, a cascade of different events and the release of several characteristic cytokines and chemokines occurs. Studies have shown that, for PAMAM dendrimers in $\mathrm{HaCaT}$ cells, an immortalized human keratinocyte line, activation of caspases 8 and 3 occurs at around 1-2 hrs, followed by mitochondrial membrane potential decay (MMPD) (3-4hrs), after which there is a release of TNF- $\alpha$ (4-5hrs) and eventually IL-8 which then maximises at 24 hours. ${ }^{38}$ It has also been proposed that larger generation dendrimer nanoparticles and/or doses can cause the endosome to rupture (endosomolysis), via the so-called proton pump mechanism..$^{39,40,41}$ Supported by the fact that after $\sim 16 \mathrm{hrs}$ the PAMAM dendrimers have been seen to be located in the mitochondria, ${ }^{22,38}$ whereupon the oxidative stress is further increased, resulting in further MMPD, which can culminate in apoptosis. A similar inflammatory cascade has been reported in murine macrophages, although the evolution of the responses was observed to be somewhat slower. ${ }^{17}$ 
In both cases, the temporal profile was seen to be systematically dependent on dendrimer generation and dose, pointing towards the basis of potential structure property relationships. However, more than a single measurement at a single time point by a cytotoxic assay, the measurement of the time evolution of the cascade provides a clearer picture of the set of events and the visualisation of different timescales allows for a better evaluation of differences due to nanoparticle properties and cell lines.

It is proposed that, by measuring and numerically modelling the kinetics of the cellular responses and their dependence on nanoparticle properties, a more intuitive formulism of the nanotoxic response which more clearly tracks the cellular response related to nanoparticle properties can be established. The approach lends itself naturally to an AOP formalism, but the systems biology like approach adds quantification of the rates of response, which are related to the physicochemical properties of the nanoparticles, such that the approach may ultimately lead to QSARs and predictive models of cytotoxic response. These predictive models would be able to provide some preliminary data on toxicity which could be used to inform initial testing strategies.

The model presented in this study looks at PAMAM toxicity based on previously published experimental data. ${ }^{16,17,23,35,38}$ A preliminary form of the model faithfully reproduced the time course of the toxic responses of the HaCaT cell-line to PAMAM dendrimer exposure, including the experimentally observed generation dependence. ${ }^{38}$ This study critically extends the model to the dose dependent responses of cell lines to nanoparticle exposure, and elucidates the origin of the dependence of $\mathrm{EC}_{50}$ on cell-type and cytotoxic assay dependent results. The approach argues for a classification of toxic responses based on activity of the particles, expressed as particle and cell dependent reaction rates, rather than Effective Concentration end points. 


\section{$\underline{\text { Materials and Methods }}$}

Experimental results are derived and adapted from previous work by the authors ${ }^{16,17,23,35,38}$ and details of the materials and methods used can be viewed in these publications. However, below is a brief summary of the experimental methods employed.

Naha et al. detail the dose and generation dependence of the toxic responses of murine macrophages, the J774A.1 cell line, to exposure to PAMAM generations G4, G5 and G6. The J774A.1 cells were cultured in Dublecco's Modified Eagle's Medium (DMEM), supplemented with $10 \% \mathrm{FBS}, 2 \mathrm{mM}$ L-Glutamine and $45 \mathrm{IU} / \mathrm{mL}$ streptomycin and penicillin at $37^{\circ} \mathrm{C}$ in $5 \% \mathrm{CO}_{2}$. The following exposure dose ranges were used: $0.08 \mu \mathrm{M}$ to $6 \mu \mathrm{M}$ for $\mathrm{G} 4,0.03 \mu \mathrm{M}$ to $2 \mu \mathrm{M}$ for $\mathrm{G} 5$ and $0.013 \mu \mathrm{M}$ to $1 \mu \mathrm{M}$ for G6. For all generations, the dose dependence of ROS production was monitored by the fluorimetric assay, Carboxy- $\mathrm{H}_{2}$ DCFDA and was performed in black 96 well plates with a cell concentration of $4 \times 10^{5}$ cells $/ \mathrm{mL}$. DMEM was used as a negative control and $\mathrm{H}_{2} \mathrm{O}_{2}$ was used as positive control. Readings were taken at $1 \mathrm{hr}, 2 \mathrm{hrs}$, $4 \mathrm{hrs}$ and $6 \mathrm{hrs}$, with $\lambda_{\text {ex }}$ $($ excitation wavelength $)=485 \mathrm{~nm}$ and $\lambda_{\text {em }}$ (emission wavelength $)=520 \mathrm{~nm}$. The temporal evolution of the inflammatory cascade of TNF- $\alpha$ and Interleucin 6 (IL-6) was monitored at a fixed dose of $1 \mu \mathrm{M}$ using the ELISA assay, where LPS was used as a positive control to verify the procedure and the absorbance was read at $405 \mathrm{~nm}$. Cytotoxicity was measured using the MTT assay, with $0.5 \mathrm{mg} / \mathrm{mL}$ dye concentration and readings were take for time points: $6 \mathrm{hrs}, 12 \mathrm{hrs}$, $24 \mathrm{hrs}, 48 \mathrm{hrs}$ and $72 \mathrm{hrs}$, at an absorbance of $595 \mathrm{~nm} .^{17,38}$

Mukherjee et al. detail the dose and generation dependences of the toxic responses of the immortalised human keratinocyte (HaCaT) and primary adenocarcinoma colon (SW480) cell lines. The cell lines were cultured in the same conditions as described above using DMEM F12 HAM and with an additional $1 \mu \mathrm{g} / \mathrm{mL}$ hydrocortisone added for the HaCaT cells. The following 
exposure dose ranges were used: $0.01 \mu \mathrm{M}$ to $21.1 \mu \mathrm{M}$ for $\mathrm{G} 4,0.03 \mu \mathrm{M}$ to $5.2 \mu \mathrm{M}$ for $\mathrm{G} 5$ and $0.01 \mu \mathrm{M}$ to $5.168 \mu \mathrm{M}$ for G6. Dose and generation dependent cytotoxicity for both cell lies was measured using the MTT, Alamar Blue and Neutral Red assays at 24hrs. The MTT assay was performed with $5 \mathrm{mg} / \mathrm{mL}$ dye concentration and read at an absorbance of $595 \mathrm{~nm}$. Alamar Blue and Neutral Red were performed in the same 96 well plates which were seeded at a concentration of $1 \times 10^{5}$ cells $/ \mathrm{mL}$. Alamar Blue was performed with a concentration of $5 \%[\mathrm{v} / \mathrm{v}]$ and results were measured using a fluorimeter with the $\lambda_{\mathrm{ex}}=485 \mathrm{~nm}$ and $\lambda_{\mathrm{em}}=520 \mathrm{~nm}$. Neutral Red was performed with a dye concentration of $1.25 \%[\mathrm{v} / \mathrm{v}]$ and results were measured using a fluorimeter with the $\lambda_{\mathrm{ex}}=531 \mathrm{~nm}$ and $\lambda \mathrm{e}_{\mathrm{m}}=642 \mathrm{~nm}$. The temporal evolutions of ROS, apoptosis and DNA damage were monitored for the $\mathrm{HaCaT}$ cell line. ROS was again measured with the Carboxy- $\mathrm{H}_{2} \mathrm{DCFDA}$ and was performed with a cell concentration of $1 \times 10^{5}$ cells $/ \mathrm{mL}$. Readings were taken between 1 4hrs and at 24hrs for HaCaT cells, and between 30mins-6hrs and at 24hrs for SW480 cells with $\lambda_{\mathrm{ex}}=490 \mathrm{~nm}$ and $\lambda_{\mathrm{em}}=545 \mathrm{~nm}$. Apoptosis was measured with flow cytometry, using the YOPRO-1/Propidium dyes at a concentration $1 \mu \mathrm{L}$ per $1 \times 10^{6}$ cells $/ \mathrm{mL}$. The TUNEL assay was used to detect DNA damage at a time point of $24 \mathrm{hrs} .^{16,23}$

Numerical results for all equations were obtained by integration using the iterative Euler approach $^{42}$ and SigmaPlot ${ }^{\mathrm{TM}}$ (v.10.0) was used to generate the values and graphs. 


\section{$\underline{\text { Results }}$}

\section{Generation of the Equations}

In order to better visualise the full range of dose and temporal dependencies and elucidate the mechanisms of response, the system was modelled using a relatively simple phenomenological rate-equation model. This model is similar, in concept, to ones commonly employed for modelling molecular-photodynamics. ${ }^{43}$

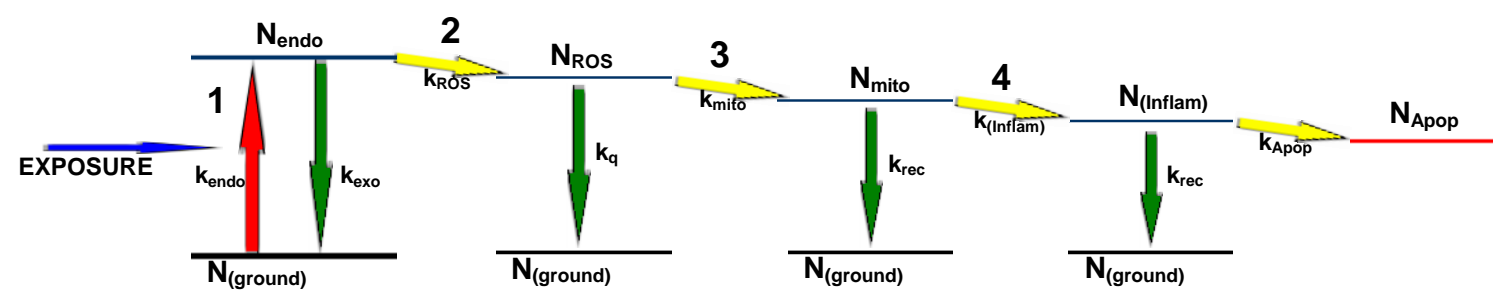

$\underline{\text { Scheme 1. Schematic of systematic sequence of events upon nanoparticle uptake. }}$

A schematic representation of the sequence of events upon nanoparticle uptake, where the cell (system) takes up a particle, progresses to ROS damage (quenched by anti-oxidants), then onto mitochondrial damage, inflammatory cascade and finally apoptosis (if the cellular recovery rates are insufficient).

Scheme 1 illustrates the systematic sequence of events which can occur when a nanoparticle is taken into a mammalian cell. 1: Upon exposure, the cells take up nanoparticles at a rate $k_{\text {endo, }}$, leading to two populations: cells without nanoparticles (i.e. in a ground/non-toxic state), $N_{\text {ground }}$, and cells with an endocytosed nanoparticle $N_{\text {endo }}$. Also indicated is a possible "exocytosis" process, occurring at a rate $k_{\text {exo }}$. However, Salvati et al. have indicated that this does not occur for 
polymeric nanoparticles and therefore it is not included in the equations. ${ }^{14} 2$ : The next step in the sequence is ROS generation at a rate $k_{R O S}$, again generating a new population $N_{R O S}$. The ROS can then be quenched by the action of antioxidants, at a rate denoted here by $k_{q}$. 3: Alternatively, the system progresses to mitochondrial damage at a rate $k_{m i t o} .4$ : This process continues through a cascade and can culminate in apoptosis if the cellular recovery rates $\left(k_{r e c}\right)$ are not sufficient.

The complex system illustrated schematically in Scheme 1 can be better described using a series of rate equations. The process of nanoparticle endocytosis by cells in vitro, taking into account the cell replication rate $\left(k_{\lambda}\right)$, has previously been described by Salvati et al. ${ }^{14}$ and is here described by Equation 2. As the dose $(D)$ is expressed as the molar dose, for ease of comparison with the experimental data, $N_{\text {endo }}$ is an expression of the molar quantity (number per unit volume) of endocytosed dendrimer nanoparticles. In subsequent equations, the term " $\mathrm{N}$ " is used to denote the equivalent quantity denoted by the subscript text that follows.

$\frac{\mathrm{dN}_{\text {endo }}}{\mathrm{dt}}=\mathrm{k}_{\text {endo }} \cdot\left(\mathrm{N}_{\mathrm{amg}}\right)^{\mathrm{c}} \cdot \mathrm{D}-\mathrm{k}_{\lambda} \cdot \mathrm{N}_{\text {endo }}(\mathrm{t})$ Equation (2)

where $k_{\text {endo }}$ is the rate of endocytosis (in units of inverse time), $N_{a m g}$ is the number of amino groups per particle, $c$ is an empirically determined fit factor, $k_{\lambda}$ accounts for cellular replication and $D$ is the molar dose. Assuming a cell duplication half life of $24 \mathrm{hrs}, k_{\lambda}$ has a value of $(0.69 / 24) \mathrm{hr}^{-1}$.

After endocytosis, nanoparticles generate ROS and this build-up of ROS is counteracted by the intrinsic intracellular anti-oxidants, in this study exemplified by GSH, and the interaction quenches both the levels of ROS and GSH. These processes have previously been described by Equations 3 and $4 .^{38}$ 
$\frac{\mathrm{dN}_{\mathrm{ROS}}}{\mathrm{dt}}=\mathrm{k}_{\mathrm{ROS}} \cdot \mathrm{G} \cdot \mathrm{N}_{\mathrm{endo}}(\mathrm{t})-\mathrm{k}_{\mathrm{q}} \cdot \mathrm{N}_{\mathrm{ROS}}(\mathrm{t}) \cdot \mathrm{N}_{\mathrm{GSH}}(\mathrm{t})$

Equation (3)

$\frac{\mathrm{dN}_{\mathrm{GSH}}}{\mathrm{dt}}=\mathrm{k}_{\mathrm{GSH}}-\mathrm{N}_{\mathrm{ROS}}(\mathrm{t}) \cdot \mathrm{N}_{\mathrm{GSH}}(\mathrm{t}) \cdot \mathrm{k}_{\mathrm{q}}$

Equation (4)

The first term in Equation 3 is a generation $(G)$ and dose $(D)$ dependent term describing continuous ROS generation at a rate $k_{R O S}\left(N_{R O S}(0)=0\right)$. The second term describes the quenching of the ROS at a rate $k_{q}$, and depends on both; ROS levels, $N_{R O S}$, and antioxidant levels, $N_{G S H}$ $\left(N_{G S H}(0)=0\right)$. In the study by Mukerjee et al., the antioxidant levels were represented by the experimentally measured values of Glutathione (GSH). ${ }^{38}$ In Equation 4, the experimentally observed linear increase of the control levels of GSH, at a rate of $k_{G S H}$, is described by the firstterm, and the second-term, which is the same as in Equation 3, describes the quenching of the GSH levels. Values of $k_{G S H}$ and $k_{q}$ are derived from the experimental data and simulations of Mukherjee et al. ${ }^{38}$

The subsequent, experimentally observed, cascade of cellular responses can be similarly simulated. The cascade elements examined were: caspase activation, mitochondrial membrane potential decay (MMPD), tumour necrosis factor alpha (TNF- $\alpha$ ) and interleukin-8 (IL-8) for HaCaT cells, or interleukin-6 (IL-6) for mouse macrophages. In the model of Mukherjee et al., ${ }^{38}$ the early stage caspase activation and MMPD are both a result of increased levels of ROS, although through independent pathways. Thus:

$\frac{\mathrm{dN}_{\text {Casp }}}{\mathrm{dt}}=\mathrm{k}_{\text {Casp }} \cdot \mathrm{N}_{\mathrm{ROS}}(\mathrm{t})-\mathrm{k}_{\text {Casp } 2} \cdot \mathrm{N}_{\text {Casp }}(\mathrm{t})$ Equation (5) 
$\frac{\mathrm{dN}_{\mathrm{MMP}}}{\mathrm{dt}}=\mathrm{k}_{\mathrm{MMP}} \cdot \mathrm{N}_{\mathrm{ROS}}(\mathrm{t})-\mathrm{k}_{\mathrm{TNF}} \cdot \mathrm{N}_{\mathrm{MMP}}(\mathrm{t})$

Equation (6)

$\frac{\mathrm{dN}_{\mathrm{TNF}}}{\mathrm{dt}}=\mathrm{k}_{\mathrm{TNF}} \cdot \mathrm{N}_{\mathrm{MMP}}(\mathrm{t})-\mathrm{k}_{\mathrm{IL}-8} \cdot \mathrm{N}_{\mathrm{TNF}}(\mathrm{t})$

Equation (7)

$\frac{\mathrm{dN}_{\mathrm{IL}-8}}{\mathrm{dt}}=\mathrm{k}_{\mathrm{IL}-8} \cdot \mathrm{N}_{\mathrm{TNF}}(\mathrm{t})$

Equation (8)

Again, in Equations 5 to $8 ; N$ describes the respective populations at time (t) and $k$ the respective rates and the initial conditions for all cases are such that $N(0)=0$. Overall, these equations show a possible cascade of events involving; ROS production, parallel rather than sequential processes of caspase activation, loss of MMP, the latter leading to activation of TNF- $\alpha$ and IL-8, as described for $\mathrm{HaCaT}$ cells exposed to PAMAM dendrimers by Mukherjee et al. ${ }^{38}$

Cell viability was monitored using the MTT assay. ${ }^{16,17}$ MTT measures the mitochondrial activity and is thus experimentally most associated with changes in the mitochondrial membrane potential, loss of which can, at certain levels, lead to apoptosis. The following equation was used to calculate the change in the population of viable cells, $N_{V}$ :

$$
\frac{\mathrm{dN}_{\mathrm{V}}}{\mathrm{dt}}=\mathrm{k}_{\mathrm{Rec}} \cdot \mathrm{N}_{\mathrm{MMP}}(\mathrm{t})^{\mathrm{b}} \cdot \mathrm{N}_{\mathrm{V}}(\mathrm{t})-\mathrm{k}_{\mathrm{V}} \cdot \mathrm{N}_{\mathrm{MMP}}(\mathrm{t}) \cdot \mathrm{N}_{\mathrm{V}}(\mathrm{t})
$$

Equation (9)

The second term of Equation 9 describes the process of cell death, dependent on the change in the mitochondrial membrane potential, and the number of viable cells. As not all cells would undergo apoptosis as a result of loss of MMP, the first term of Equation 9 allows for a process of cell recovery, at a rate $k_{R e c}$, and an empirically determined sub-linear dependence (b) on $N_{M M P}$ 
makes this process more prominent at lower doses. $N_{V}$ is initially set equal to a value of 1 to represent $100 \%$ cellular viability.

The phenomenological rate equation model thus enables simulation of the time evolution of the cellular responses as well as the measured cytotoxic response.

Notably, the only particle dependent parameters are the nanoparticle dose $(D)$ and generation $(G)$. It is demonstrated that the model can reproduce the experimentally observed variations of cytotoxic response as a function of dose and generation, as well as measurement time-point and the dependence on cell-line parameters such as intrinsic intracellular antioxidant levels.

\section{$\underline{\text { Temporal Evolution }}$}

For a fixed dose and generation, the temporal evolution of the cellular responses has previously been faithfully simulated using the rate equation model with the HaCaT cell-line. ${ }^{38}$ The model can be extended to the J774A.1 cell-line, for G4 exposure at a fixed dose of $1 \mu \mathrm{M}$, as shown in Figure 1 (experimental data reproduced from Naha, P.C. et al, $2010^{17}$ ). Experimental data (as symbols) is shown only for ROS and TNF- $\alpha$ as well as the temporal dependence of the MTT viability assay (as symbols and dotted line as a guide to the eye). The solid red line indicates the simulated ROS production and the other dotted lines show the temporal evolution of: Caspases (Green), MMPD (Orange) and TNF- $\alpha$ (Purple) as simulated according to Equations 3 to 8. For both experimental and simulated data, results are normalised to a maximum value of 1 for visualisation purposes, with the exception of the MTT data (viability - blue dotted line), which experimentally have been normalized to the unexposed control ${ }^{17}$. Parameters employed for the simulated data of Figure 1, and for all subsequent figures, are listed in Supplemental Information, Table S1. The sensitivities of the temporal, dose and generation dependences of the simulated 
data to variations of these parameters are analysed in the Discussions and Conclusions section, and, where appropriate, in the Supplemental Information.

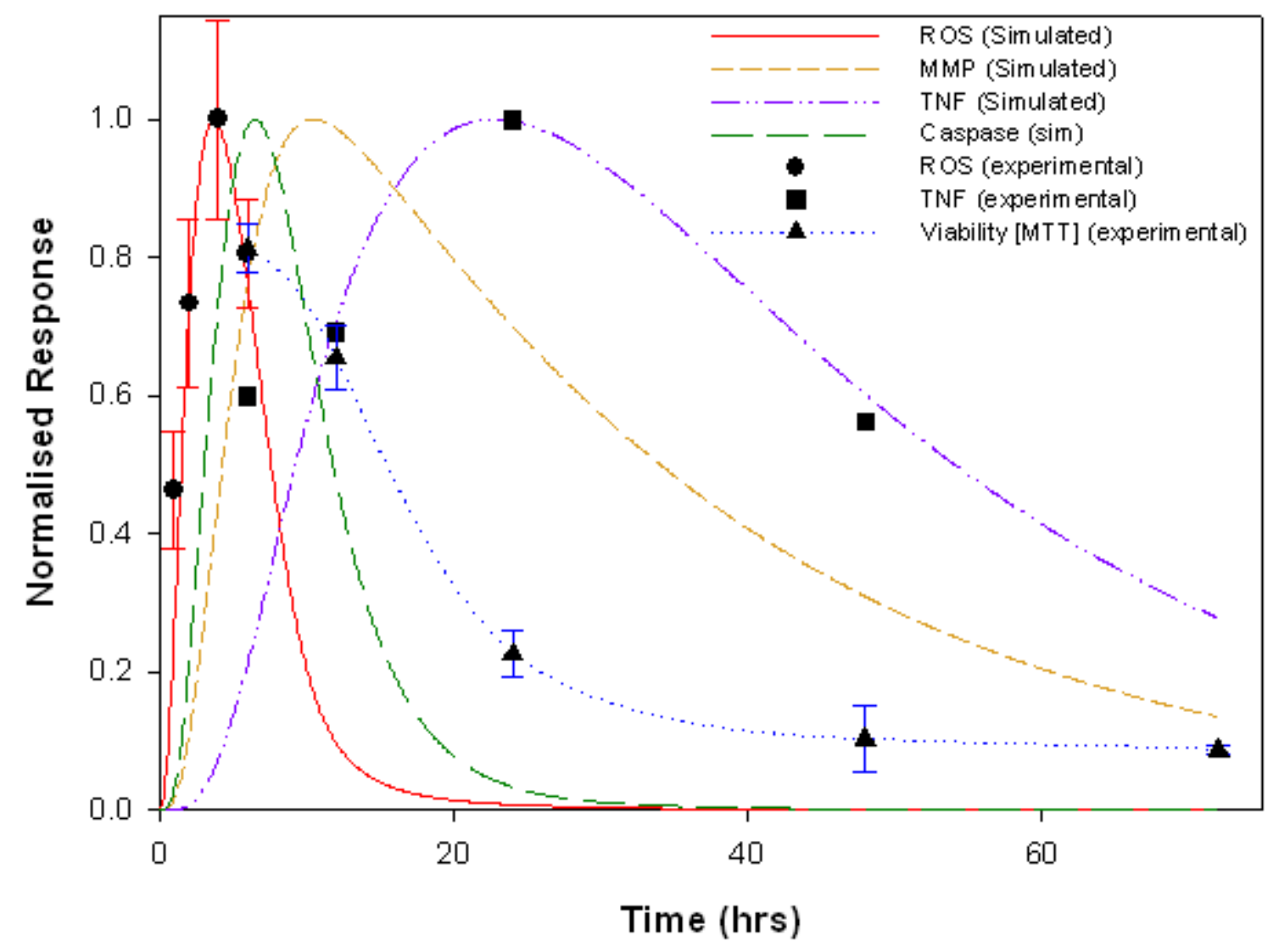

Figure 1. Experimental (from Naha, P.C. et al, $2010^{17}$ ) and simulated cellular responses of J744A.1 murine macrophages to exposure to G4 PAMAM dendrimers at a fixed dose of $1 \mu \mathrm{M}$. $\underline{\text { Results are normalised to a maximum value of } 1 \text { for visualisation purposes, with the exception of }}$ the MTT data (viability), which experimentally have been normalized to the unexposed control.

\section{$\underline{\text { ROS Dose Dependence }}$}

The rate equation model can therefore be applied to simulate the temporal evolution of the cellular responses to exposure to PAMAM dendrimers at a fixed dose. Furthermore, in the 
previous study by Mukherjee et al., the generation dependences of Equations 2 and 3 were seen to faithfully reproduce the experimentally observed more rapid onset of ROS production with increasing dendrimer generation. ${ }^{38}$ Notably, however, using a constant ROS generation rate, $k_{R O S}$, the ROS levels were found to increase monotonically as a function of time, and it was found necessary to constrain the rate of generation to decrease, dependent on the amount of ROS generated, indicative of a depletion of the source of ROS. In the preliminary model of Mukherjee et $a l,{ }^{38}$ this was achieved through the relationship of Equation 10.

$\frac{\mathrm{dk}_{\mathrm{ROS}}}{\mathrm{dt}}=-\mathrm{k}_{\mathrm{ROS}} \cdot \mathrm{N}_{\mathrm{ROS}}(\mathrm{t})$ Equation (10)

While this approach allowed the faithful reproduction of the temporal evolution of the ROS levels, it does not accurately model the complex dose/generation dependence of the ROS levels at a fixed time point, as shown in Supplementary Information Figure S1. Figure 2 shows the dose and generation dependent levels of ROS in the J774A.1 cell-line, expressed as the percentage increase in Carboxy-H2DCFDA fluorescence compared to control, at a fixed timepoint of 4hrs (data derived from Naha, P.C. et al, $2010^{17}$ ).

The dose dependence of Figure 2 can however be reproduced by considering that the increased levels of ROS are the result of an interaction of the endocytosed nanoparticles with an intracellular source of ROS, $N_{\text {source }}$, which is depleted by the ROS generation process. Thus, $\frac{\mathrm{dN}_{\text {Source }}}{\mathrm{dt}}=-\mathrm{k}_{\mathrm{A}} \cdot \mathrm{G} * \cdot\left(\mathrm{N}_{\text {endo }}(\mathrm{t})^{\mathrm{A}}\right) \cdot\left(\mathrm{N}_{\text {Source }}(\mathrm{t})^{\mathrm{B}}\right)$ Equation (11) 
where $k_{A}$ is the interaction rate for the nanoparticles and source, $G^{*}$ is a generation dependant factor and the values for $A$ and $B$ are empirical constants. The generation of ROS, previously described by Equation 3, is then described by:

$\frac{\mathrm{dN}_{\mathrm{ROS}}}{\mathrm{dt}}=\mathrm{k}_{\mathrm{A}} \cdot \mathrm{G} *\left(\mathrm{~N}_{\text {endo }}(\mathrm{t})^{\mathrm{A}}\right) \cdot\left(\mathrm{N}_{\text {Source }}(\mathrm{t})^{\mathrm{B}}\right)-\mathrm{k}_{\mathrm{q}} \cdot \mathrm{N}_{\mathrm{ROS}}(\mathrm{t}) \cdot \mathrm{N}_{\mathrm{GSH}}(\mathrm{t})$

Equation (12)

The continuous lines of Figure 2 show the simulation of the dose and generation dependent increase in ROS in the J774A.1 cell-line, as calculated at 4hrs, in comparison to the experimentally observed values. The simulated data are expressed in arbitrary units, and have been scaled to give a best match to the experimental data. Experimentally, a background level of $\sim 10 \%$ was observed at even the lowest doses and thus the simulated data, which begins at a value of zero, are offset for comparison.

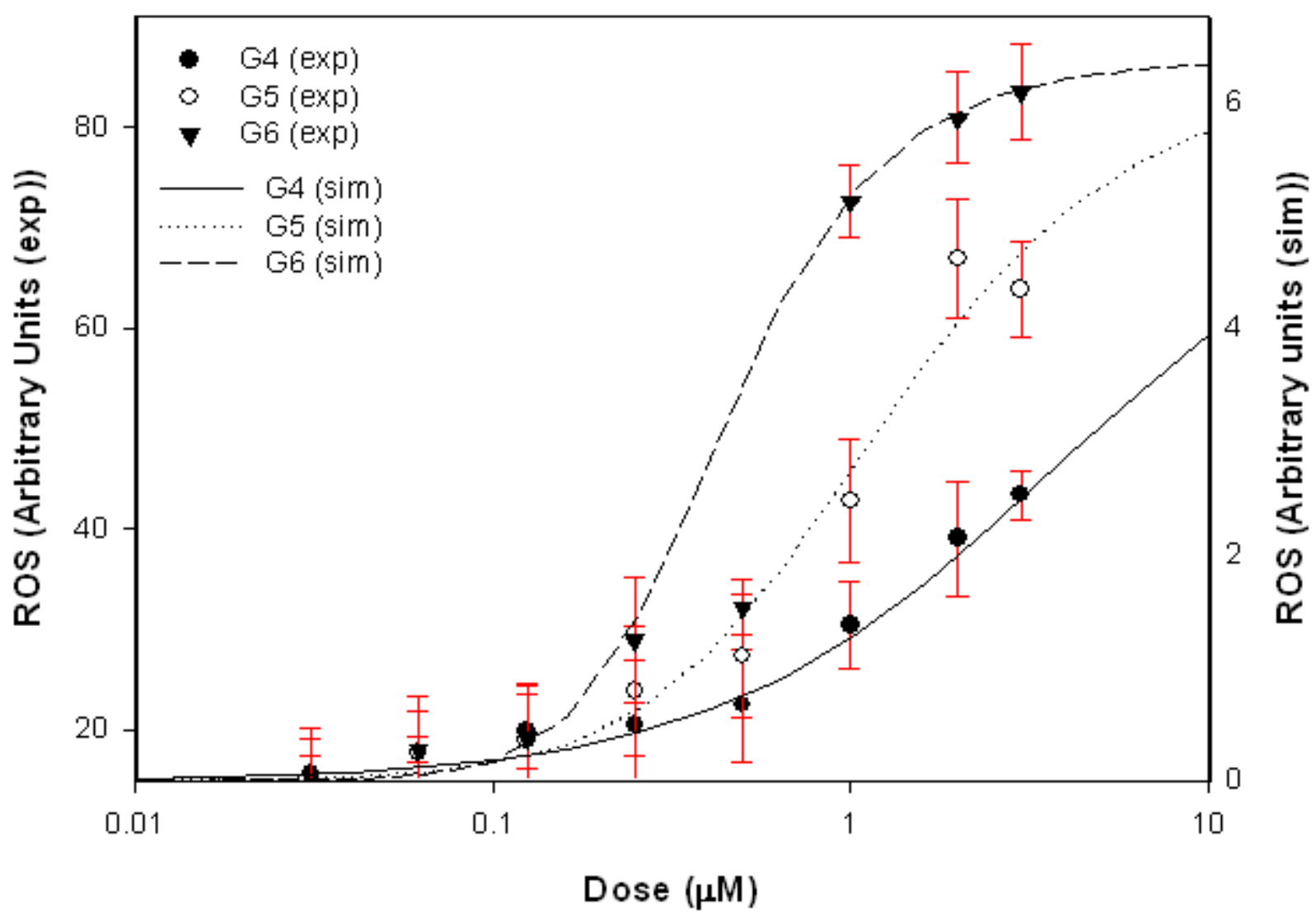


Figure 2. Experimentally observed (symbols) (expressed as the \% increase in Carboxy-

$\underline{\mathrm{H}}_{2} \underline{\text { DCFDA fluorescence compared to control, at a fixed timepoint of } 4 \mathrm{hrs}}{ }^{17}$ ) and simulated

(lines) dose and generation dependent intracellular ROS generation after $4 \mathrm{hr}$ exposure of

J744A.1 mouse macrophages to PAMAM dendrimers.

The simulated data of Figure 2 are generated from Equations 1-14, simultaneously for all three dendrimer generations, with only $G$ and $D$ as variables. The closest simulated reproduction of the experimental data was achieved using a generation dependent expression of $G^{*}$ and $A$, with a constant value of $B=3$, such that:

$\mathrm{G}^{*}=\left(\frac{1}{\mathrm{~N}_{\mathrm{amg}}^{0.25}}\right)$

$\mathrm{A}=\left(\frac{\mathrm{N}_{\mathrm{amg}}}{64}\right)^{0.75}$

Note, in the simulated data of Figure 2, a value of 0.25 was used for " c" of Equation 2. The initial value of $N_{\text {source }}(t=0)$ was chosen to be $10 \mu \mathrm{M}$, the maximum dose level used in the simulation.

The simulation faithfully reproduces the dose and generation (number of surface amino groups) dependence of the production of ROS. Notably, for each generation, the maximum levels of ROS production saturate at the same value, consistent with experimental observations supporting the proposition that the surface amino groups are the primary source of the response to the nanoparticle intake. ${ }^{24}$ The evolution of the equations (from Equation 10 to Equations 11 and 12) to account for the dose dependence of the ROS response has little effect on the temporal profiles 
of the cellular responses at a fixed dose, and the responses represented in figure 1 are calculated using the latter formalism rather than that of Equations 3 and 4.

Experimentally, the evaluation of the dose (7 dose measurements) and generation ( 3 generations) dependent increases in ROS levels as well as their temporal evolution (1-24hrs) is an extensive series of measurements, in independent triplicates. Having established a model which can faithfully simulate the responses of the cell line, a 3-D representation of the combined and continuous dose dependence and temporal evolution can be constructed, as shown in figure 3 for the case of G4. Similar 3-D representations can be constructed for G5 and G6, and the model can be readily extrapolated to higher and lower generations. Although the 2-D representation of the 3-D system does not do it justice, the view illustrates the importance of considering the3-D plane in the system response. 


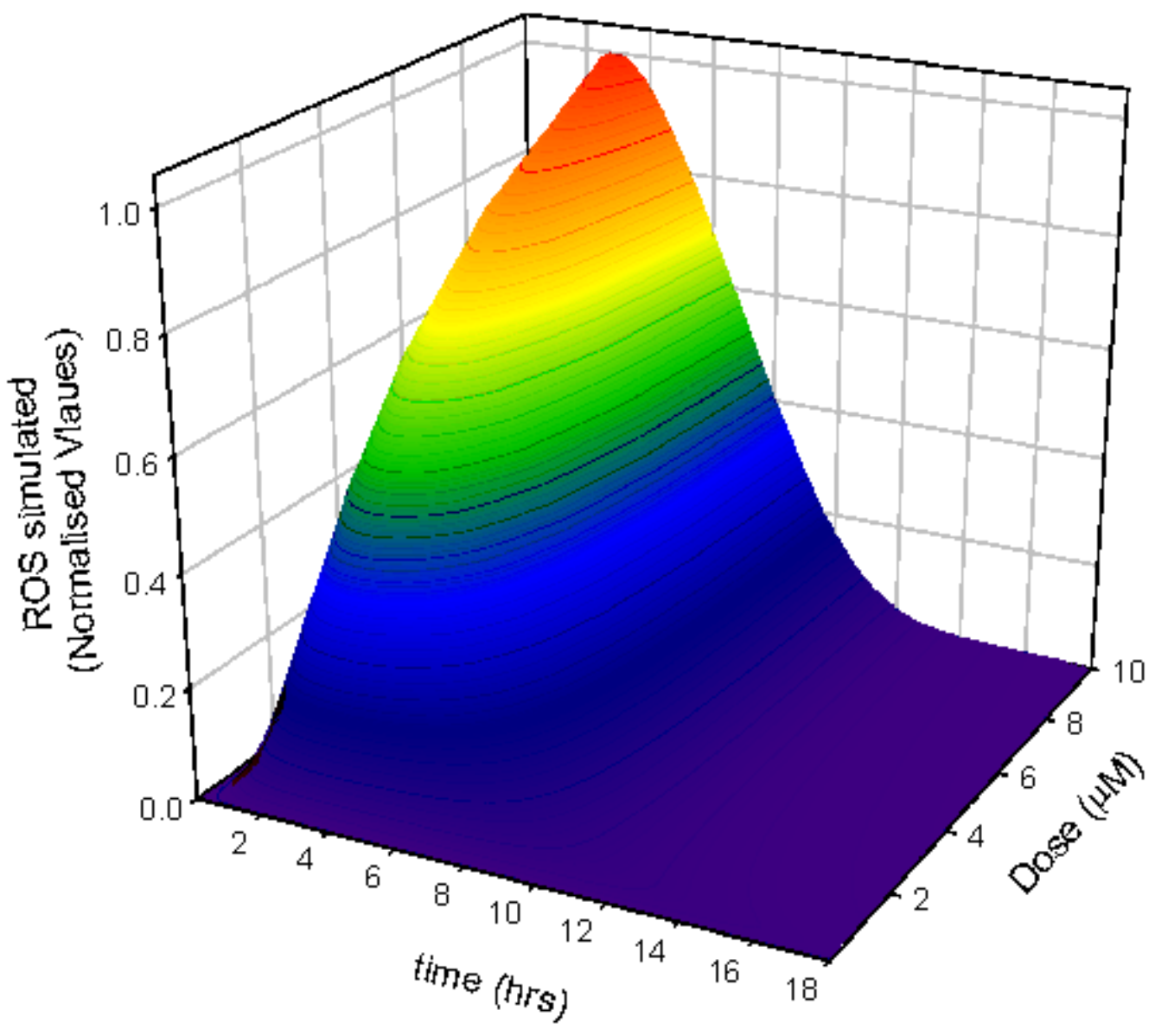

Figure 3. A 3D graph showing both the simulated time and dose dependence of the ROS evolution, in J744A.1 mouse macrophages, upon G4 PAMAM nanoparticle uptake.

\section{Cellular Viability}

It is clear that the approach of the phenomenological rate equation model can accurately reproduce the experimentally observed cellular responses, as a function of dose and generation, as well as their temporal evolution. For the given cell-line, the responses are determined by the surface reactivity of the nanoparticles, which, in the case of PAMAM dendrimers presented here, depends on the number of surface amino groups per particle for each generation. Ultimately, the 
response to nanoparticle exposure is reduced viability in the in vitro culture, as measured by cytotoxicological assays such as MTT, and normally represented by the $\mathrm{EC}_{50}$. It has previously been shown that the cytotoxicity of PAMAM dendrimers is systematically dependent on generation, but, given the complex dose and temporal responses, the exact link to nanoparticle structure and reaction mechanisms has not been elucidated.

Figure 1 also plots the experimentally observed $\mathrm{MTT} \mathrm{EC}_{50}$ value at different time points (from Naha, P.C. et al, $2010^{17}$ ). For a given generation, the dose dependence of the reduction in cellular viability at different timepoints can be simulated according to Equation 9. Figure 4 illustrates the simulated dose dependence of the cellular viability, for a single generation (G4), at $6 \mathrm{hrs}, 12 \mathrm{hrs}$ and $24 \mathrm{hrs}$, compared to the corresponding experimentally observed MTT response at these timepoints (from Naha, P.C. et al, $2010^{17}$ ). All parameters used to simulate the dose dependence of the ROS (figure 2) as well as the temporal evolution of the cellular responses (figure 1) are kept constant and only the dose is varied. A value of 0.25 was employed for the parameter b in Equation 9. The model, with reasonable accuracy, reproduces the in vitro cellular responses observed experimentally. The simulated results are best matched to the experimental responses at the earlier (6hr) and later (24hr) timepoints. The deviation from the observed responses at the intermediate timepoint (12hrs) may be the result, for example, of more complex quenching mechanisms not included in the model at this stage. 


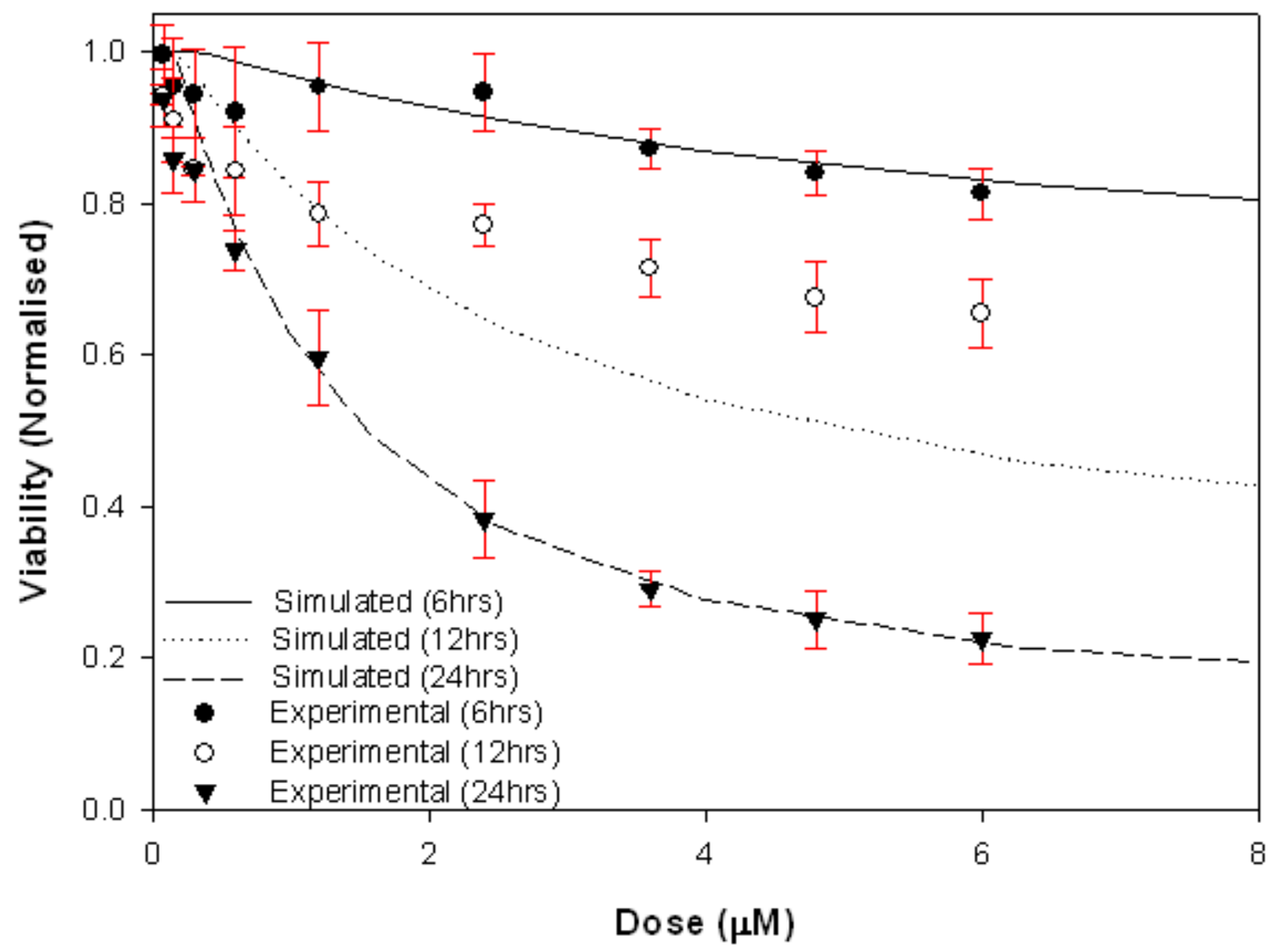

Figure 4. Experimental (symbols) and simulated (lines) dose dependent viability, as measured using the MTT assay, for J744A.1 murine macrophages at $6 \mathrm{hr}, 12 \mathrm{hr}$ and $24 \mathrm{hr}$ (experimental data from Naha, P.C. et al, $2010^{17}$ ).

By varying the generation $(G)$, for a fixed time point, the dose dependence of the cellular viability for each generation can be similarly qualitatively reproduced, as shown in figure 5 , for the case of $12 \mathrm{hr}$ exposure of mouse macrophages (experimental data derived from Naha, P.C. et al, $\left.2010^{17}\right)$. 


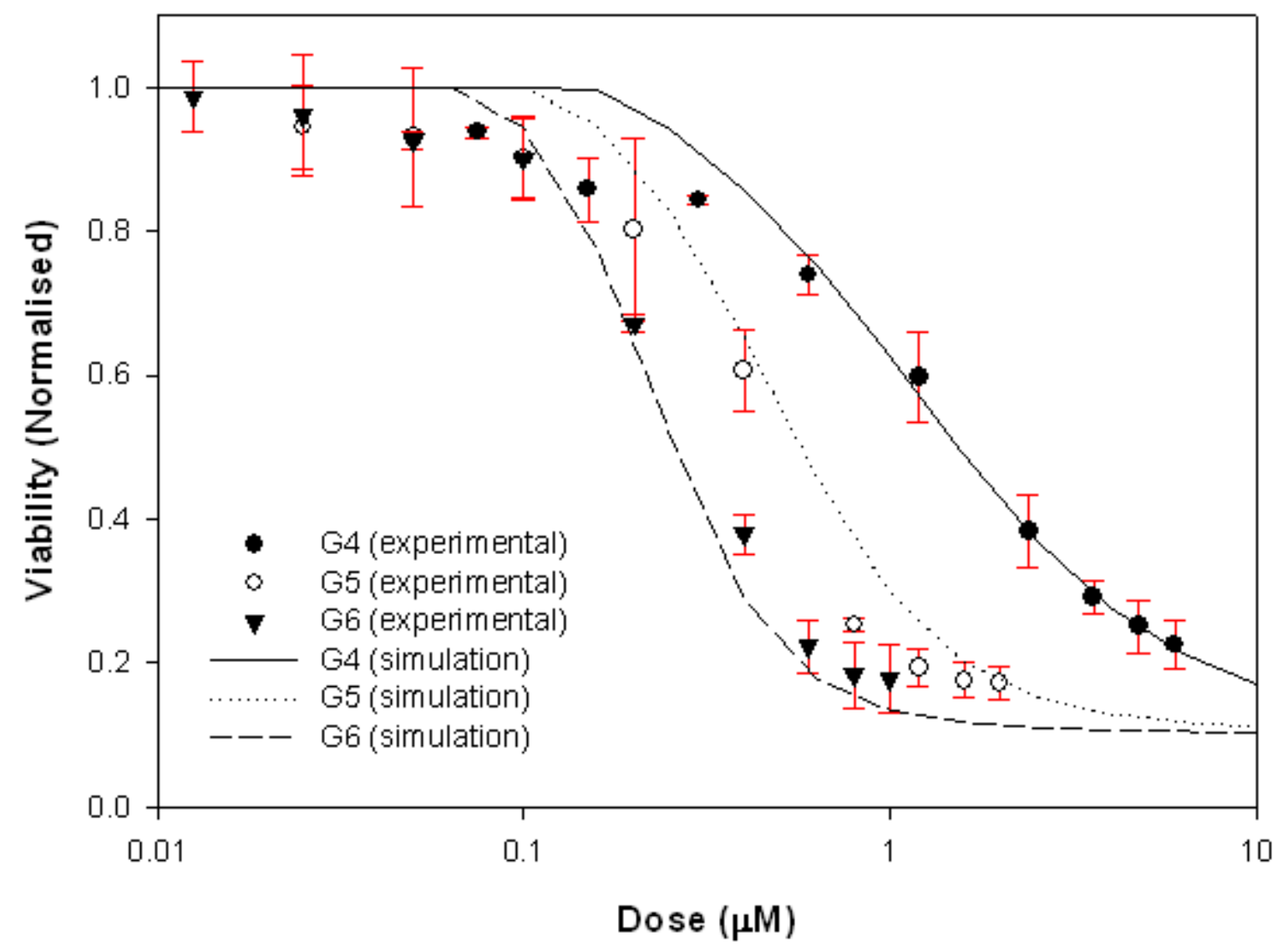

Figure 5. Experimental (symbols) and simulated (lines) dose dependent J744A.1 mouse macrophage viability at $12 \mathrm{hrs,}$, as measured using the MTT assay, for PAMAM generations G4, G5 and G6 (experimental data from Naha, P.C. et al, $2010^{17}$ ).

Similar to the case of the ROS, a 3-D representation of the simulated cytotoxic response of HaCaT cells to PAMAM dendrimer exposure, as a function of dose and time, can be constructed, as shown in Figure 6 for the case of G4. A similar representation can be made for G5 and G6, and the model is readily extendable to higher or lower PAMAM generations. 


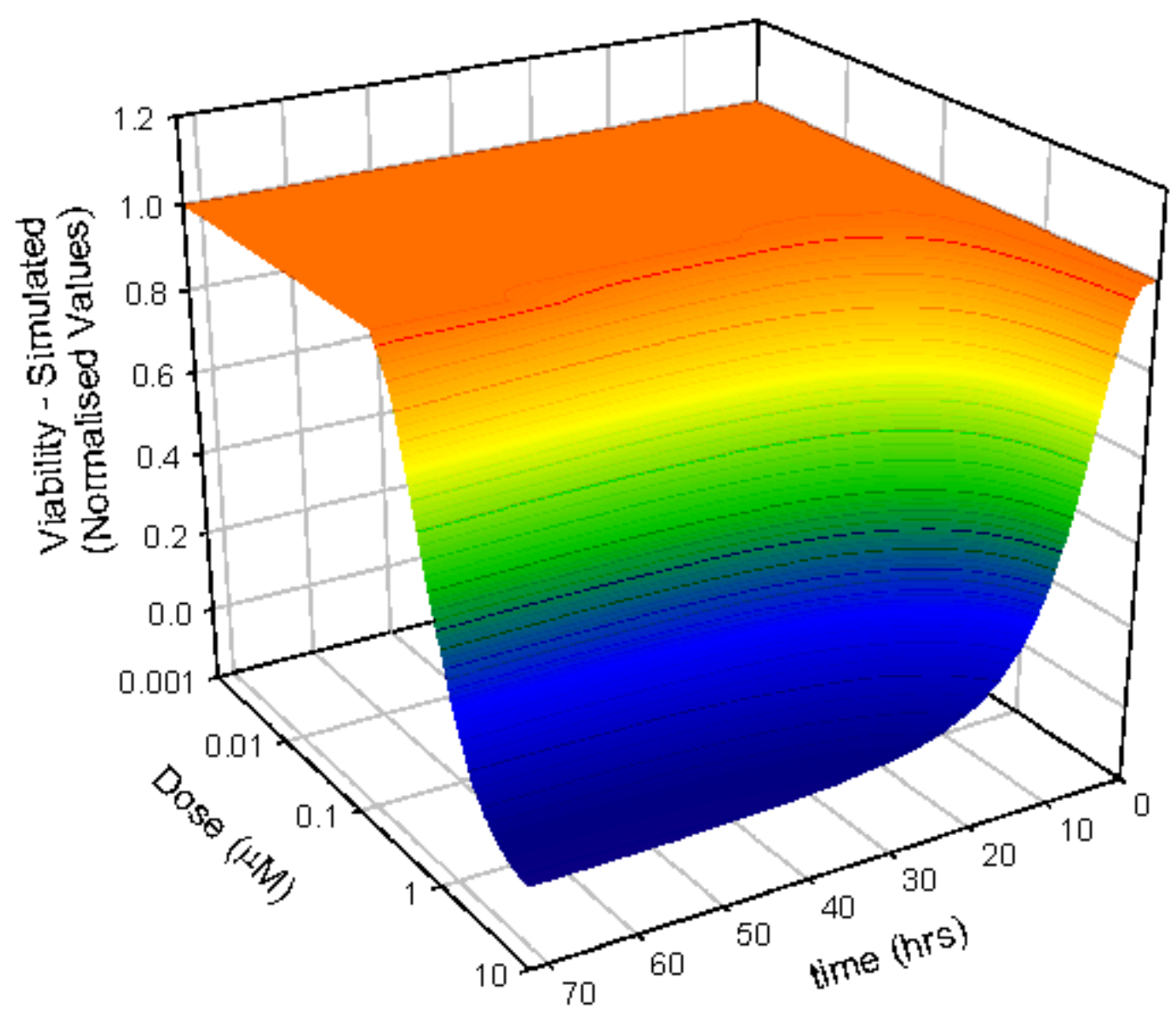

Figure 6. 3D simulated time and dose dependence of MTT response for exposure of J744A.1 murine macrophage cells to PAMAM G4.

The experimental data of Figure 5 is a typical representation of a cytotoxicological screening of nanoparticles. In comparing the responses of the cell line to the different generations in the dendrimer series, a systematic structural dependence of the response is clear. The application of the model presented here demonstrates that that dependence is derived from the number of surface amino groups per dendrimer generation, and the rate of generation of ROS. The true value of any such model, however, depends on its applicability to a range of different experimental scenarios. ${ }^{32}$ One notable source of diversity in the literature on in vitro nanotoxicology is the range of different cell lines used, often justified as models for target organs 
in vivo. Indeed, in the work of Naha et al., and Mukherjee et al., a range of different cell lines were employed. ${ }^{16,17,23,24,38}$ In addition to the murine macrophages already described in this manuscript ${ }^{17}$, the human colon adenocarcinoma cell line (SW480) and the immortalised human keratinocyte line $(\mathrm{HaCaT})$ were employed as models of ingestion and transdermal transport of nanoparticles respectively. ${ }^{16,23,38}$ Notably, the different cell lines gave significantly different results for the cytotoxicity of PAMAM dendrimers, as shown in figure 7 for the examples of the dose dependent viability as measured using the MTT assay upon exposure of the different cell lines to PAMAM G4 for 24hrs. Although an extrapolated $\mathrm{EC}_{50}$ from the J774A. $1^{17}$ and SW480 ${ }^{16}$ cell lines would yield similar results, the value for $\mathrm{HaCaT}^{16}$ is considerably higher, indicating a greater resistance of the skin cell line to external insult, as may be expected.

\section{Variability: Cell lines and Assays}

Following the cascade of cellular responses after nanoparticle endocytosis, described in Figure 1 and represented by Equations 2-14, a potential source of the differences in cell type responses to nanoparticle exposure is the constituent levels of cellular antioxidants, redox enzymes etc., which help the cell to protect itself from the oxidative stress caused by an external agent. For example, the average activities of three important intracellular redox agents, glutathione (GSH), glutathione-S-transferase, and glutathione reductase are approximately three times higher in WI38 human fibroblast cells than in HaCaT cells and therefore, upon $24 \mathrm{~h}$ exposure to arsenic, HaCaT cells have been reported to be $50 \%$ more susceptible to cell death than WI38 cells. ${ }^{44}$ Of relevance to the study presented in figure 6, the natural intracellular glutathione (GSH) level of $\mathrm{HaCaT}$ cells are reported to be $7300 \mathrm{nmol} / \mathrm{mg}$ protein ${ }^{44}$ whereas those of SW480 and J774A.1 cells have been reported to be $\sim 40 \mathrm{nmol} / \mathrm{mg}$ protein ${ }^{45}$ and $26.7 \mathrm{nmol} / \mathrm{mg}$ protein, ${ }^{46}$ respectively. 
The higher level of antioxidants in $\mathrm{HaCaT}$ cells is therefore consistent with a greater resistance to external insult. In the rate equation approach presented here, this internal resistance to oxidative stress is represented by $k_{G S H}$ in Equation 6. The solid/dashed lines of figure 7 represent the simulated viability for the different cell lines, calculated according to Equation 12, having increased only the rate of generation of GSH for the HaCaT cell line (by a factor of 1.5), in Equation 4, keeping all other rates constant. It can be seen that the simple change in the cell dependent response parameter faithfully reproduces the experimentally observed differences in response for the different cell lines. The increased levels of GSH result in a more rapid quenching of the intracellular ROS levels, and this is manifest experimentally as an initial maximum in $\mathrm{HaCaT}$ cells after $1-2 \mathrm{hrs},{ }^{38}$ whereas in J774A.1 cells, the maximum is observed at 3-4 hrs (Figure 1). ${ }^{17}$

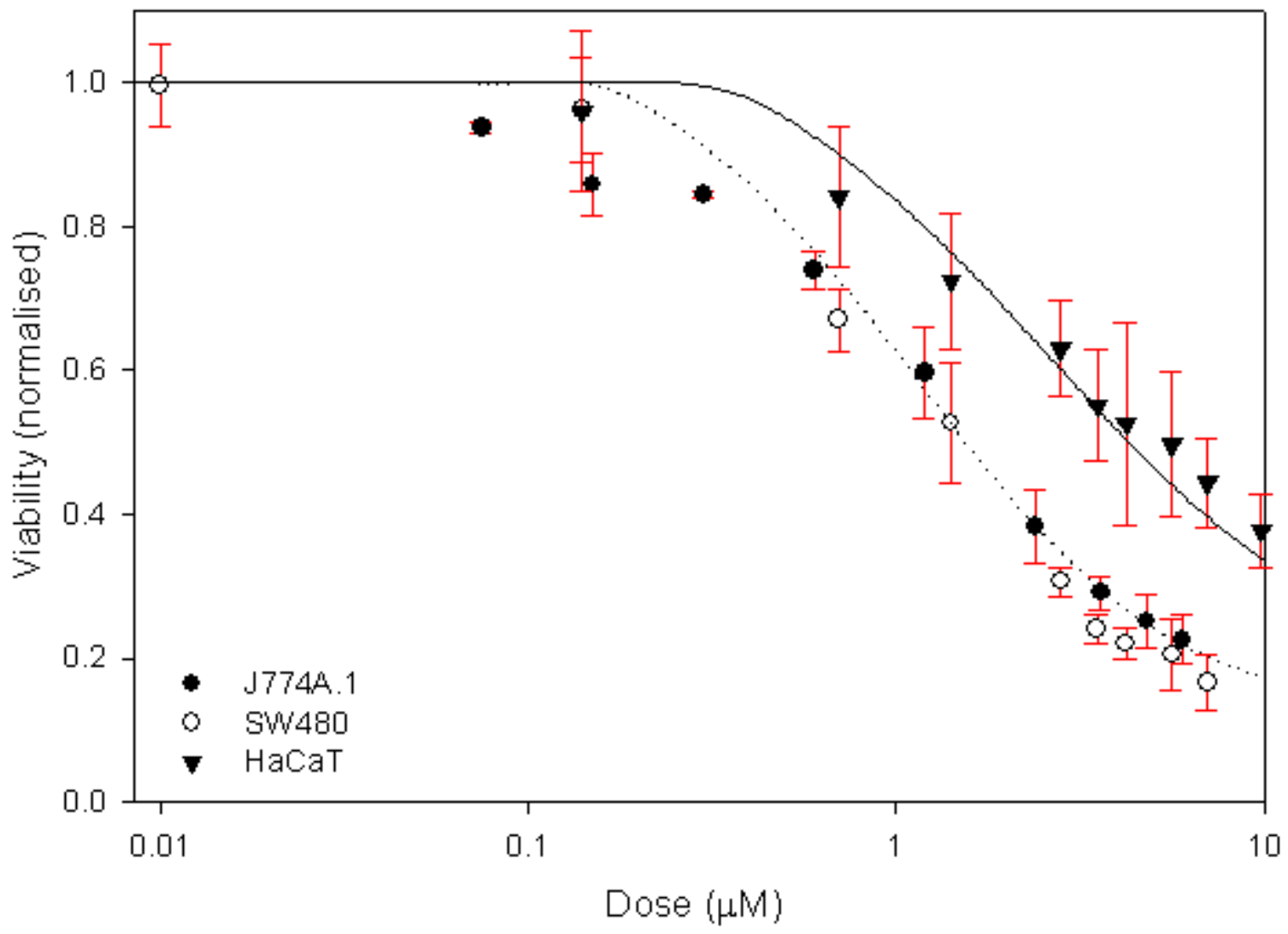


Figure 7. Experimental (symbols) and simulated (lines) viability of different cell lines, as measured using the MTT assay, after exposure to PAMAM G4 for $24 \mathrm{hrs}$. Experimental data for J774A.1 derived from Naha, P.C. et al, $2010^{17}$. Experimental data for SW480 and HaCaT derived from Mukherjee, S.P. et al. $2010^{16}$.

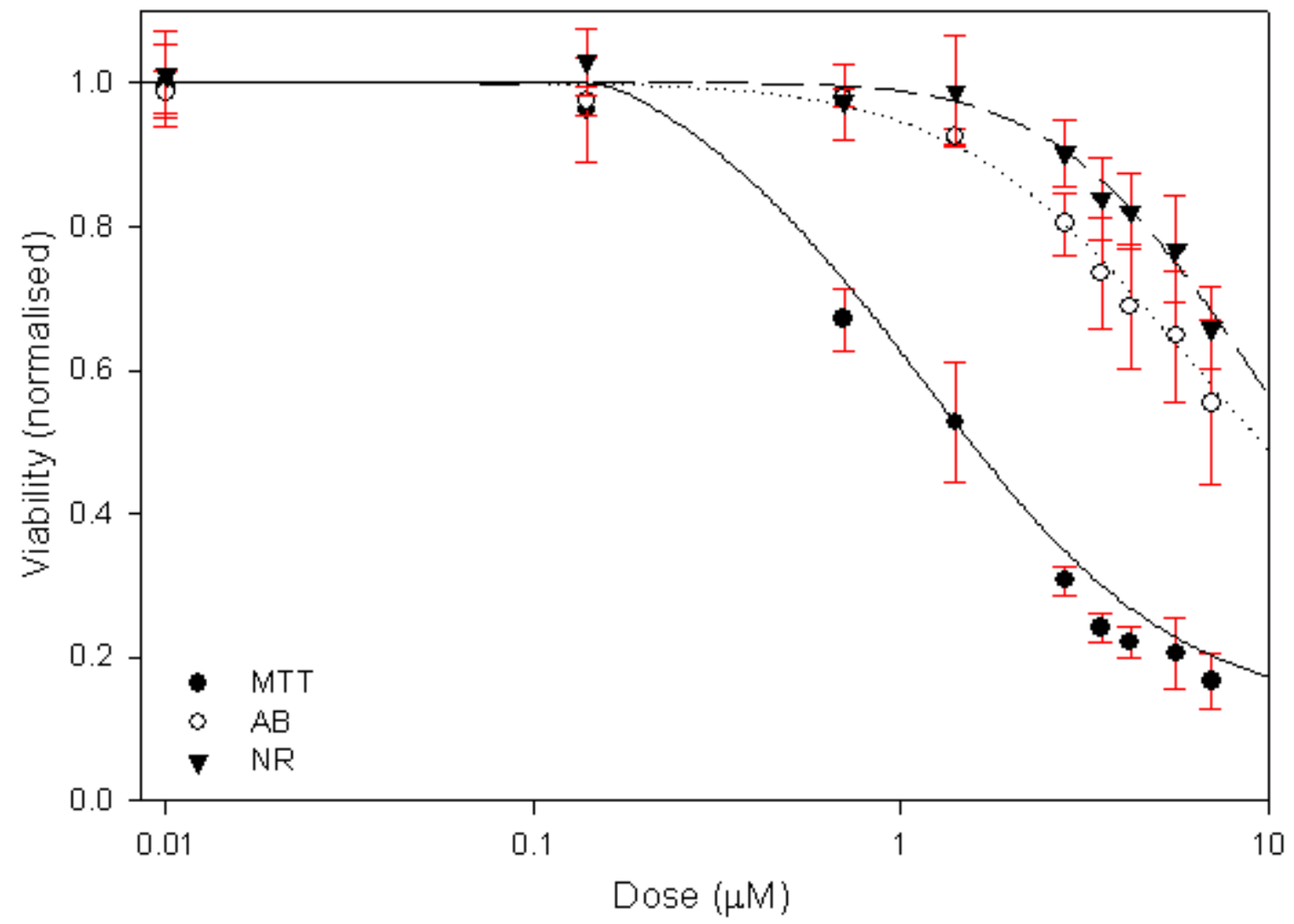

Figure 8. Comparison of Experimental (symbols) and simulated (lines) viabilities as measured by MTT, Alamar Blue and Neutral Red, for exposure of SW480 cells to PAMAM G4 after 24 hrs. Experimental data from Mukherjee, S.P. et al. $2010^{16}$. For each assay, the data has been normalized to unexposed control.

Literature furthermore describes the use of a diverse range of endpoints to evaluate the cytotoxic responses of cell lines in vitro. Each assay measures a different underlying cellular response or function. As discussed above, MTT measures the mitochondrial function. Alamar Blue is a 
fluorescence based assay which is used to assess cell viability and proliferation; this is done by measuring the innate metabolic activity via the conversion of resazurin (non-fluorescent) to resorufin (fluorescent). ${ }^{47}$ Neutral Red is another fluorometric dye, which measures the lysosomal activity using 3-Trimethyl-2,8-phenazinediamine, monohydrochloride, which binds to the lysosomes of viable cells. ${ }^{48}$ Mukherjee et al. have highlighted how the different sensitivities of the assays as a result of the exposure to PAMAM dendrimers are a manifestation of the mechanism of interaction of the nanoparticles with the cells. ${ }^{38}$ Figure 8 reproduces the experimental values of the dose dependent viability of SW480 cells after $24 \mathrm{hr}$ exposure to PAMAM G4 as measured by the MTT, Alamar Blue and Neutral Red assays (from Mukherjee, S.P. et al. $2010^{16}$ ). For each assay, the data has been normalized to unexposed control.

In the rate equation model presented here, the responses of the assays are represented by different steps in the cascade of cellular responses following exposure. The MTT response is associated with changes in mitochondrial membrane potential, and is simulated by Equation 9. The lower sensitivities of the Alamar Blue and Neutral Red assays indicate that they are associated with later responses in the cascade. The Alamar Blue response can be associated with the subsequent phase of generation of TNF- $\alpha$ and modelled according to Equation 13.

$$
\frac{\mathrm{dN}_{\mathrm{V}}{ }^{\prime}}{\mathrm{dt}}=\mathrm{k}_{\mathrm{rec}}{ }^{\prime} \cdot \mathrm{N}_{\mathrm{TNF}-\alpha}(\mathrm{t})^{\mathrm{b}} \cdot \mathrm{N}_{\mathrm{V}}{ }^{\prime}(\mathrm{t})-\mathrm{k}_{\mathrm{V}}{ }^{\prime} \cdot \mathrm{N}_{\mathrm{TNF}-\alpha}(\mathrm{t})^{\mathrm{m}} \cdot \mathrm{N}_{\mathrm{V}}{ }^{\prime}(\mathrm{t})
$$

Note that $N_{V}$ ' in Equation 13 represents the number of viable cells as registered by Alamar Blue. $N_{V}^{\prime}(0)=1$ represents the initial $100 \%$ viability. In modelling the experimental data of Figure 8 , for Alamar Blue, it is noted that an empirical factor of $m=2$ provides a good fit and a value of $b$ $=0.25$ was again used. 
In a similar fashion, the response of Neutral Red can be associated with a later stage of the cascade of cellular responses and simulated according to Equation 14:

$\frac{\mathrm{dN}_{\mathrm{V}}{ }^{\prime \prime}}{\mathrm{dt}}=\mathrm{k}_{\mathrm{rec}}{ }^{\prime \prime} \cdot \mathrm{N}_{\mathrm{IL}-8}(\mathrm{t})^{\mathrm{b}} \cdot \mathrm{N}_{\mathrm{V}}{ }^{\prime \prime}(\mathrm{t})-\mathrm{k}_{\mathrm{V}}{ }^{\prime \prime} \cdot \mathrm{N}_{\mathrm{IL}-8}(\mathrm{t})^{\mathrm{p}} \cdot \mathrm{N}_{\mathrm{V}}{ }^{\prime \prime}(\mathrm{t})$

$N_{V}{ }^{\prime \prime}(0)=1$ represents the initial $100 \%$ viability. A good reproduction of the experimental results is found with an empirical factor of $p=3$ and $b=0.25$. In Equations (13) and (14), $k_{\text {rec }}$ ' and $k_{\text {rec }}$,', and $k_{V}$ ' and $k_{V}$ ', describe the rates of recovery of the system without apoptosis, and the rates of change of the viability, as measured by the respective assay. 


\section{$\underline{\text { Discussion }}$}

The phenomenological rate equation model presented here is a demonstration of how an integrated approach to analysis of the responses of cells to nanoparticle exposure can be employed to provide a better visualisation of the dependences of those responses on experimental parameters such as dose and exposure time, nanoparticle characteristics (such as number of active surface groups) and even variations in cell line and cytotoxicity assay. The model is constructed on previous experimental observations of the cellular responses to PAMAM dendrimers, which have systematically variable physico-chemical properties, and have been seen to elicit systematically varied cellular responses. The mechanisms of response and their causal nature and inter-relationships have been explored and discussed extensively elsewhere. ${ }^{16,17,23,38}$ While it is impractical at this stage to cover all such potential cellular responses, the model is used to simulate several known cytotoxic markers, simultaneously for all 3 dendrimer generations, and thus is at least a partial system of events. Such a system of events can be considered as a sub-section of an Adverse Outcome Pathway ${ }^{49,31}$ and is consistent with the approach of predictive paradigms for assessing nanotoxicological responses. ${ }^{50,33}$

The initial stage of endocytosis has previously been described using a rate equation approach for the uptake of 40-50 nm fluorescently labelled polystyrene nanoparticles in the A549 human lung adenocarcinoma cell line, as measured by flow cytometry. ${ }^{14}$ Endocytosis rates were measured and simulated according to build up of cellular fluorescence, and the values of $k_{\text {endo }}$ in the current model were chosen to yield similar simulated results for the build up of dendrimer nanoparticles in the cells, as shown in supplementary material Figure S2. In contrast to the fluorescently labelled PS nanoparticles, the dendrimers are not easily visualized within the cells and so the cellular response of increased levels of ROS was used to guide the simulation of the time, dose 
and generation dependence of the cellular responses. Figure $\mathrm{S} 4$ shows the sensitivity of the simulated dose dependent ROS generation to variations in the parameter $k_{\text {endo. }}$. In the current model, the best simulations were achieved by including a generation $\left(N_{\text {amg }}\right)$ dependent factor in the uptake, as described in Equation 2, with a $c$ value of 0.25. For comparison, Supplementary Figure S4 compares the dose dependent generation of ROS after 4hrs exposure for all 3 generations, with varying values of the parameter $c$. The best replication of the response for all generations simultaneously is achieved using a value of $c=0.25$. In understanding the potential significance of this factor, it is worth observing that the relationship between the diameter and the number of surface amino groups for PAMAM dendrimers in the region of $N_{a m g}=20-1000$ is a power law of order 0.25 (data from http://www.dendritech.com/pamam.html). The nominal diameters of PAMAM dendrimers available on the dendritech website are in good agreement with those measured by size exclusion chromatography. ${ }^{51}$ The values of hydro-dynamic diameter measured by Dynamic Light Scattering in PBS are somewhat larger, as may be expected, but the values still scale systematically with generation and although a slight increase in cell culture medium due to interaction with the medium may be inferred, no significant aggregation/agglomeration of the particles is evident over the dose ranges measured. ${ }^{16}$ Thus, a dependence of the rate of endocytosis on $N_{\text {amg }}{ }^{0.25}$ may represent a dependence on the dendrimer diameter, which varies only slightly with generation number (G4 diameter: $4.5 \mathrm{~nm}$, G5 diameter: 5.4nm and G6 diameter: $6.7 \mathrm{~nm}^{16}$ ).

The model considers the case of PAMAM dendrimers which have been demonstrated to be taken up in mammalian cell lines by a process of endocytosis. ${ }^{14}$ However, endocytosis can be subcategorised into four different types: Clathrin-mediated endocytosis, Macropinocytosis, Caveolae-mediated endocytosis and Phagocytosis, all of which could be readily modelled via 
modification of the rate equation approach used in this study. It is also important to note that, for smaller generation dendrimers, a potential for passive uptake is also possible. It would be of great interest to continue to reduce the dendrimer size and explore the transition between the dominance of active and passive uptake mechanisms, which could be incorporated as a parallel uptake process using such a rate equation approach.

While a more primitive version of the model presented here was capable of reproducing the temporal evolution of the cellular responses, and to some extent the dependence on dendrimer generation, ${ }^{38}$ an accurate model of the complex dose dependence of the increase in intracellular ROS is key to extending the model to simulate the dose dependent cytotoxic responses.

The intracellular levels of ROS increase upon nanoparticle endocytosis but are seen to reach a maximum on a time scale of 1-4 hours, depending on the dendrimer generation, dose and cell line. ${ }^{17,38}$ In the rate equation formulation presented here, this transient behaviour cannot be reproduced simply by introduction of an anti-oxidant quenching mechanism. Rather, a limit to the capacity of the cell to generate increased levels of ROS is inferred. This process is represented by the reaction of the endocytosed nanoparticles with a source species, which generates ROS, while consuming the source. The approach enables faithful simulation of both the temporal evolution and the dose and generation dependence of the ROS.

The approach is analogous to that of the Operational Model of Agonist Activation, ${ }^{52}$ which invoked the concept of an agonist receptor, to which the agonist binds, to produce the response. Using the law of Mass Action at equilibrium, a faithful reproduction of a sigmoidal response curve to a linear agonist dose was achieved, based on the agonist concentration, the receptor concentration and the agonist/receptor dissociation rate. 
In considering the biological significance of an ROS source, $N_{\text {source }}$, understanding the mechanisms of ROS generation is important and has been discussed in the supplementary information (ROS source discussion).

Although the exact process of ROS production has not been experimentally visualised, the model can still faithfully reproduce the dose and generation dependence of the increase in ROS levels, using Equations 11 and 12, as shown in Figure 2. All curves of Figure 2 can be modelled with a constant value of $k_{A}$. Figure S5 of the supplementary information illustrates how the simulated dose dependent increase in the ROS levels for all three generations at a fixed timepoint of $4 \mathrm{hrs}$ depends on variation of the parameter $k_{A}$. This value is normalised by the factor $G^{*}=N_{\text {amg }}{ }^{-0.25}$. The sensitivity of the simulations to the exponent of this parameter is shown in Figure S6. Notably, this is the inverse of the factor employed for Equation 2, and, in the light of the discussion above, can be related to the generation dependence of the dendrimer diameter.

For a given dendrimer generation, the rate of generation of ROS is dependent on $N_{\text {endo }}(t)^{A} \cdot N_{\text {Source }}(t)^{B}$. As the dose $(D)$ is expressed as the molar dose, for ease of comparison with the experimental data, $N_{\text {endo }}$ is an expression of the molar quantity of endocytosed dendrimer nanoparticles. Figures S7 and S8 demonstrate the sensitivity of the simulated dose dependent ROS generation on the parameters $A$ and $B$ respectively. The dependence of the power law factor, $A$, on the number of amino groups, $N_{a m g}$, is a reflection of the increasing reactivity per dendrimer nanoparticle with generation. The value of $B=3$ indicates that, in the reaction process, 3 source species are involved.

In understanding the influence of the different parameters of Equation 12 on the form of the dose dependent ROS generation of Figure 2, and by analogy to the Operational Model of Black and 
Leff, ${ }^{52} k_{A} G^{*}$ is largely responsible for the $\mathrm{EC}_{50}$ of the curves, while the factors $A$ and $B$ govern the Hill slope, $A$ accounting for the observed dendrimer generation dependence.

It is however important to note that for these well defined structures, the nanoparticle diameter, number of surface amino groups, and surface area all scale with generation number and are therefore intimately related. It has also been previously demonstrated that the (positive) zeta potential scales with increasing generation and therefore surface area and number of surface amino groups $N_{a m g}{ }^{16}$. In cell culture medium, the zeta potential for all generations becomes negative due to interaction with the medium and scales with the generation, surface area and therefore $N_{a m g}$, and notably, the magnitude of the change in the zeta potential scales with the dendrimer surface area. For both the SW480 and HaCaT cell line, the $\mathrm{EC}_{50}$ for a range of assays (Neutral Red, Alamar Blue, MTT (all 24hrs) and the clonogenic assay ( 8 day)) has been seen to scale with the surface area, the zeta potential in cell culture medium, and the change in zeta potential from distilled water to cell culture medium. Zeta potential depends on the dispersion environment, however, and is not an intrinsic nanoparticle parameter, compared to the number of surface amino groups, which is compositionally defined. A similar systematic correlation between the toxic response and the number of surface amino groups has been demonstrated for J774A.1 mouse macrophages. ${ }^{17}$

Using only $G$ as a nanoparticle dependent variable, resulting in a reaction rate which is dependent on the systematically varied physico-chemical structure of the dendrimer nanoparticles, the experimentally observed dose and time dependent ROS generation can be simultaneously simulated for all three dendrimer generations. Using this as the initiation stage of an intracellular response cascade, the subsequent changes in viability of the cell population at different timepoints, as measured using the MTT assay, can be modelled simultaneously using the 
relatively simple rate Equation 9. As MTT primarily monitors changes in mitochondrial activity, changes in cell viability are related to the time dependent MMPD, as simulated by Equation 6 . The sensitivity of the simulations to the parameter $k_{M M P}$ of Equation 6 is shown in Supplementary Information Figure S9. It is noted that, using this relatively simple formalism, an accurate simultaneous reproduction of the experimental results at all timepoints is not achieved. This is not surprising as the cascade process of cytokine activation/production and resultant changes to mitochondrial activity is more complex than that considered here. The first term of Equation 9 allows for changes in MMP without reduction of cell viability as measured by the MTT assay, but this did not significantly improve the simultaneous simulation of the dose dependent viability at different time points.

Similarly, Equation 9 can be employed to simultaneously simulate the cytotoxicity for all three generations, at a fixed timepoint. In figure 5, the simulation provides an accurate reproduction of the experimental data, although the low dose behaviour is less well reproduced. Again, this is most likely a result of the relative simplicity of the model system, which doubtless does not include many processes which are occurring in parallel with those experimentally measured and considered in the model. Figure S10 of the Supplementary Information explores the sensitivity of the simulated viability to the parameter $k_{M M P}$. No significant improvement in the discrepancy at low doses is achieved, although the simulated curves of Figure 5 fall largely within the experimental error margins.

A potential pitfall of such modelling approaches can be 'over parameterisation' with insufficient experimental data that ultimately leads to 'over fitting'. In modelling the system, the minimum number of sequential processes have been included to describe the system, as observed 
experimentally. Additional steps in the cascade can be added, as they are elucidated experimentally or by for example pathway enrichment approaches.

It should be noted that the ROS response to PAMAM nanoparticle exposure has been observed to be a two stage response, ${ }^{38}$ and only the initial stages are modelled here. The initial response (0$6 \mathrm{hrs}$ ) has been associated with encapsulation of the dendrimers in endosomes, while the later, secondary response has been associated with release of the nanoparticles into the cytoplasm by endosomolysis and subsequent incorporation into the mitochondria. A similar behaviour was observed by Xia et al. $(2006)^{12}$ for ROS generation by aminated polystyrene nanoparticles. While the second phase of ROS generation is not modelled here, a similar rate-equation approach could be employed to simulate the responses, their time evolution and dependences on dose and dendrimer generation. A more complete understanding of generation and dose dependence of the process of endosomal-rupture, particle migration to and uptake by the mitochondria, and recovery of intracellular antioxidant levels would be required. Between the two phases, such phenomena as the quenching of ROS levels to below those of controls, the recovery of antioxidant levels, and migration of antioxidants to localised subcellular sites should be considered. Nevertheless, the phenomenological model is readily adaptable to include more complex phenomena, simply by adding additional terms to the rate-equations. However, it should be noted that while the ROS generation of figure 2 relates exclusively to the early stages of intracellular nanoparticle interactions, the later responses, in particular the later time point viability measurements, may be influenced by the secondary phases of ROS generation.

Nevertheless, the model provides a valuable visualisation of the range of cellular responses to the exposure to nanoparticles and a faithful qualitative reproduction of the nanoparticle structural and dose dependences of the cytotoxic responses based on independent nanoparticle and cell 
dependent reaction rates. Notably, experimentally observed differences between the responses of different cell lines can be simulated simply by adjustment of cellular passed parameters, in this case the rate of production of GSH, based on established differences in cell-line metabolism. In this context, the robustness of the model can be further validated by monitoring variations of ROS production and cytotoxic responses as a result of externally controlled antioxidant levels. ${ }^{13,53,54,55,56 \text { reviewed by } 57}$

Furthermore, the model also elucidates the origin in the differences of the responses of commonly used cytotoxicity assays and clarifies that lower observed values of $\mathrm{EC}_{50}$ for different assays as a measure of the same nanoparticle/cell-line system are an indication that the assays are registering cellular responses at later stage in the response cascade. Although the time responses have not explicitly been measured, the simulations of Figure 8 indicate that the variations of responses can be well modelled by Equations 13 and 14, which have the same form as the viability simulations of Equation 9, keeping all parameters of the earlier stage responses constant. Figure S11 shows the variability of the fits to changes in the parameters $m$ and $p$.

The use of such rate equation models for the simulation or fitting of complex systems is by no means novel and has become more prevalent in pharmacokinetics/pharmacodynamics. ${ }^{58,59} \mathrm{In}$ terms of nanobio interactions, Salvati et al. employed a rate equation approach to model the uptake of polystyrene nanoparticles in cells as measured by the fluorescent response of the cell populations, although no modelling of the cellular responses was undertaken. ${ }^{14}$ Dell'Orco et al . used a similar approach, described in terms of Systems Biology, to model the delivery success rate of engineered nanoparticles and its dependence on the presence and structure of the protein corona. ${ }^{60}$ To our knowledge, however, this is the first use of such models to simulate the time evolution, dose and structural dependence of cellular responses to nanoparticle exposure and to 
derive toxicological endpoints from the simulated data. Undoubtedly, the approach is crude at this stage, and improvements based on fitting of the data by for example nonlinear regression ${ }^{59}$ or parameter global sensitivity analysis $(\mathrm{GSA})^{60}$ could significantly enhance the robustness of the model. Parameter GSA simultaneously varies all the parameters within the parameter space to evaluate their effect on the system. ${ }^{60}$ Program packages are available to aid this analysis, such as: SBML-SAT (Systems biology markup language - sensitivity analysis tool). This program allows for evaluation using a range of different methods, including Sobol's Method, Weighted average of local sensitivity analysis, multi-parametric sensitivity analysis and partial rank correlation coefficient. The program can also handle discontinuous events, which could be beneficial for the system in question. ${ }^{61}$ While this sort of modeling shows potential for the future it is worthy to note that uncertainty still remains an issue. For example a study by Bennett et al, shows that when calculating one parameter using a range of different models, the results can be orders of magnitude in difference. ${ }^{62}$ Differences will always be present in these models as different models utilize different methods for prediction. However, the scale of the differences should be reduced, particularly by larger validation sets and more detailed applicability domains.

Nevertheless, the model helps to visualise the complex range of responses which can occur upon exposure of in vitro cell cultures to nanoparticles. The experimentally observed cascade of events resulting from oxidative stress and subsequent inflammatory responses can readily be modelled. The dose dependence is complex, but can be modelled and related to the nanoparticle physicochemical properties. Critically, the model demonstrates the temporal responses are dynamic and the dose dependences are complex, and therefore that it is not sufficient to measure cellular responses at a fixed dose or time point. Ideally, the complete 3-D profile of time, dose and response should be measured for all particles, structural variations, assays and cell-lines. Such an 
exercise would be exhaustive, in terms of time and cost, however, and numerical simulation models such as the one presented here can be of great benefit in reducing the effort.

Notably, the model allows to differentiate the impact on the viability endpoint of nanoparticle based parameters from cellular based parameters, and for a single cell line, the entire range of responses to the exposure to three different dendritic nanoparticles has been reduced to the two parameters of rate of endocytosis and rate of ROS generation. Within a single cell line, the differing responses of the different cytotoxicity assays are consistent with sensitivity to different stages of the cellular response cascade. Extending to different cell-lines, the variation of response can be accounted for by differences in intracellular antioxidant levels, although cell-line dependent differences in rate of endocytosis and rate of ROS generation, for the same nanoparticles cannot be ruled out and need to be experimentally verified.

The representation of the cellular responses in terms of a causally related sequence of events leading to cell death lends itself naturally to an AOP formalism, and such a formalism can be advantageous to regulatory bodies as it will identify the critical step in the sequence leading to a toxic response, in this case ROS production. Although AOPs developed to-date are primarily qualitative in nature, the work described in this manuscript could be described as modelling a 'partial' AOP or Toxicity Pathway that comprises events at the molecular and cellular level. Additionally, the systems biology like approach adds quantification of the rates of response, which are related in the initial stages to the physico-chemical properties of the nanoparticles. By coupling these two approaches it may be possible to calculate an amount or rate of ROS production, from which the cell cannot recover, which would form the cut off point (acceptable limit) of whether the nanoparticle would be considered dangerous or not. Of course cellular quenching of ROS via antioxidant activity would also have to be considered. Such an approach 
may ultimately lead to predictive models of cytotoxic response and the basis of QSARs. In terms of the development of QSARs, the study and modelling of homologous series of nanoparticles contributes greatly to interpreting the mechanism of response, but also quantifiably relating the endpoint $\left(\mathrm{EC}_{50}\right)$ to the nanoparticle physico-chemical properties. The model further indicates, at least within the homologous series presented here, that the determining factor of the toxic response is the rate of generation of ROS, and that this may be considered a defined endpoint for the construction of QSARs. Obviously, the domain of applicability of such a model would need to be expanded by studies of a broader range of polymeric and other nanoparticles whose surface chemistry is well defined and ideally systematically variable. Only then can a reliable statistical validation of reliability and robustness of the model be considered. In constructing the model, simulations have been performed simultaneously for either: three dendrimer generations (Figures 2 and 5), timepoints (Figure 4), cell lines (Figure 7) or assays (Figure 8), keeping all other parameters constant. This suggests that, once the model has been established, it should be readily extendable to, for example, further generations of PAMAM dendrimer, similar homologous cationic dendrimer series such as Poly(propylene imine), or other aminated polymer nanoparticles such as polystyrene and that the responses should be quantitatively predictable based on the number of surface amino groups. Work is ongoing to demonstrate that the model can be quantitatively translated to such systems, and thereby demonstrate a broader domain of applicability. Ultimately, it may be possible to characterise the surface reactivity, and thus the rate of generation of ROS acellularly, such that the model could the readily be extended to other more complex particle types that act via ROS mediated cellular stress pathways such as metal oxides, and the parameter may be used as input into such a model to predict the in vitro cytotoxicity. 


\section{Conclusion}

The plethora of in vitro studies of nanoparticle toxicity demand that a more structured approach to classification and understanding. In this context, homologous series of particles of systematically variable physico-chemical properties play an important role, and the systematically varied cellular responses facilitate the development of mathematical models based on the mechanisms of response.

This study demonstrates how such an approach can be used to faithfully reproduce and visualise the time and dose dependant response, for three polymer dendrimer nanoparticles simultaneously, and to construct a 3D toxicity profile to model the structurally dependent nanoparticle uptake and resultant oxidative stress and inflammatory cascade, leading to apoptosis and cell death. The phenomenological rate equation approach lends itself naturally to tracking the kinetics of the response, but the dose dependent toxicological end point at a fixed time point can similarly be modelled.

The model is also readily extendable to different cell lines, simply by changing a cell based parameter such as $k_{G S H}$ and while the full range of components of the inflammatory/apoptotic cascade have not yet been experimentally visualised, the model can replicate the results of the cytotoxicity assays by solving for viability at different steps in the sequence. Furthermore, the system of equations used is versatile and can be readily modified to include additional cascade steps and new parameters as the experimental data become available.

Work is ongoing to apply the model to a range of different particles and cell lines. This current work serves to show that these mathematical models hold potential in predicting nanoparticle toxicity and will, with further development, provide a viable alternative to testing of each particle 
via a case-by-case approach. The models also have potential uses in a regulatory capacity and by combining these quantative methods with qualitative methods (such as AOPs) it will be easier to evaluate and apply limits of acceptability to the toxic response. 


\section{$\underline{\text { Author Contributions }}$}

All authors have given approval to the final version of the manuscript.

\section{Funding Sources}

Irish Government's Programme for Research in Third Level Institutions, Cycle 4, National Development Plan 2007-2013, supported by the European Union Structural Fund. M Maher is funded through the DIT Fiosraigh President's Award for Research Excellence 2010

\section{$\underline{\text { Declaration of interest }}$}

The authors declare no conflict of interest related to the work presented in this manuscript.

\section{$\underline{\text { Keywords }}$}

Nanotoxicology, Polyamidoamine Dendrimer, Reactive Oxygen Species, Inflammatory cascade, Cytotoxicity, Rate Equation Model, Numerical Simulation, Quantitative Structure Activity Relationships

\section{Acknowledgements}

This work was conducted in part under the framework of the INSPIRE programme, funded by the Irish Government's Programme for Research in Third Level Institutions, Cycle 4, National Development Plan 2007-2013, supported by the European Union Structural Fund. M Maher is funded through the DIT Fiosraigh President's Award for Research Excellence 2010.

\section{$\underline{\text { Abbreviations }}$}

ROS, reactive oxygen species. $\mathrm{EC}_{50}$, effective concentration $50 \%$. QSAR, quantative structure activity relationship. PAMAM, polyamidoamine. GSH, glutathione. MMPD, mitochondrial 
membrane depolarization. TNF- $\alpha$, tumour necrosis factor - alpha. IL-8, interleukin-eight, IL-6, interleukin 6 . 


\section{$\underline{\text { References }}$}

1. Project on Emerging Nanotechnologies (PEN), http://www.nanotechproject.org/, 2013

2. Jain, K.K. Applications of Nanobiotechnology in Clinical Diagnostics. Clinical Chemistry, 53, 2002-2009 (2007)

3. Balasundaram, G., Sato, M., Webster T.J. Using hydroxyapatite nanoparticles and decreased crystallinity to promote osteoblast adhesion similar to functionalizing with RGD. Biomaterials, 27, 2798-2805 (2006)

4. Nishimura, I., Huang, Y., Butz, F., Ogawa, T., Lin, A. and Wang, C.J. Discrete deposition of hydroxyapatite nanoparticles on a titanium implant with predisposing substrate microtopography accelerated osseointegration. Nanotechnology, 18, 1-9 (2007)

5. Nair, H.B., Sung, B., Yadav, V.R., Kannappan, R., Chaturvedi, M.M. and Aggarwal, B,B. Delivery of anti-inflammatory nutraceuticals by nanoparticles for the prevention and treatment of cancer. Biochemical Pharmacology, 80, 1833-1843 (2010)

6. Bharali, D.J., Khalil, M., Gurbuz, M., Simone, T.M., Mousa, S.A. Nanoparticles and cancer therapy: A concise review with emphasis on dendrimers. Int. J. Nanomed., 4, 1-7 (2009)

7. Koo, O.M., Rubinstein, I. and Onyuksel, H. Role of nanotechnology in targeted drug delivery and imaging: a concise review. Nanomed: Nanotech. Bio. Med., 1, 193-212 (2005)

8. Hans, M.L. and Lowman, A.M. Biodegradable nanoparticles for drug delivery and targeting. Curr. Opin. Solid St. M., 6, 319-327 (2002) 
9. Watson, P., Jones, A.T. and Stephens, D.J. Intracellular trafficking pathways and drug delivery: fluorescence imaging of living and fixed cells. Adv. Drug Deliver. Rev., 57, 43-61 (2005)

10. De Jong, W. H. and Borm, P.J.A. Drug delivery and nanoparticles: Applications and hazards. Int. J. Nanomed., 3, 133-149 (2008)

11. Jain, K.K., P. Gupta, U. and Jain, N.K. Dendrimer toxicity: Let's meet the challenge. Int. J. Pharm., 394, 122-142 (2010)

12. Xia, T., Kovochich, M., Brant, J., Hotze, M., Sempf, J., Oberley, T., Sioutas, C., Yeh, J.I., Wiesner, M.R. and Nel, A.E. Comparison of the Abilities of Ambient and Manufactured Nanoparticles to Induce Cellular Toxicity According to an Oxidative Stress Paradigm. Nano Lett., 6, 1794-1807 (2006)

13. Nel, A., Xia, T., Mädler, L. and Li N. Toxic potential of materials at the nanolevel. Science, $311,622-627(2006)$

14. Salvati, A., Aberg, C., dos Santos, T., Varela, J., Pinto, P., Lynch, I. and Dawson K.A. Experimental and theoretical comparison of intracellular import of polymeric nanoparticles and small molecules: toward models of uptake kinetics. Nanomed: Nanotech. Bio. Med., 7, 818-826 (2011)

15. Kennedy, I.M., Wilson, D. and Barakat, A.I. Uptake and Inflammatory Effects of Nanoparticles in a Human Vascular Endothelial Cell Line. HEI Research Report 136, Health Effects Institute, Boston, MA (2009) 
16. Mukherjee, S.P., Davoren, M. and Byrne H.J. In vitro mammalian cytotoxicological study of PAMAM dendrimers - towards quantitative structure activity relationships. Toxicol. in Vitro, 24, $169-177$ (2010 a)

17. Naha, P.C., Davoren, M., Lyng, F.M. and Byrne, H.J. Reactive oxygen species (ROS) induced cytokine production and cytotoxicity of PAMAM dendrimers in J774A.1 cells. Toxicol. Appl. Pharm., 246, 91-99 (2010)

18. Rothen-Rutishauser, B., Mühlfeld, C., Blank, F., Musso, C. and Gehr, P. Translocation of particles and inflammatory responses after exposure to fine particles and nanoparticles in an epithelial airway model. Part. Fibre. Toxicol., 4. doi: 10.1186/1743-8977-4-9 (2007)

19. Schins, R.P., McAlinden, A., MacNee, W., Jimenez, L.A., Ross, J.A., Guy, K., Faux, S.P. and Donaldson, K. Persistent Depletion of I Kappa B Alpha and Interleukin-8 Expression in Human Pulmonary Epithelial Cells Exposed to Quartz Particles. Toxicol. Appl. Pharm., 167, $107-117(2000)$

20. Shvedova, A.A., Castranova, V., Kisin, E.R., Schwegler-Berry, D., Murray, A.R., Gandelsman, V.Z., Maynard, A. and Baron, P. Exposure to carbon nanotube material: assessment of nanotube cytotoxicity using human keratinocyte cells. J. Tox. Env. Heal. A, 66, 1909-1926 (2003)

21. Medina, C., Santos-Martinez, M.J., Radomski, A., Corrigan, O.I. and Radomski, M.W. Nanoparticles: pharmacological and toxicological significance. Brit. J. Pharmacol., 150, 552-558 (2007) 
22. Lee, J.H., Cha, K.E., Kim, M.S., Hong, H.W., Chung, D.J., Ryu, G. and Myung, H.

Nanosized polyamidoamine (PAMAM) dendrimer-induced apoptosis mediated by mitochondrial dysfunction. Toxicol. Lett., 190, 202-207 (2009)

23. Mukherjee, S.P., Lyng, F.M., Garcia, A., Davoren, M. and Byrne H.J. Mechanistic studies of in vitro cytotoxicity of poly(amidoamine) dendrimers in mammalian cells. Toxicol. Appl.

Pharm., 248, 259-268 (2010 b)

24. Naha, P.C. and Byrne, H.J. Generation of Intracellular Reactive Oxygen Species and Genotoxicity effect to Exposure of Nanosized Polyamidoamine (PAMAM) dendrimers in PLHC1 cells in vitro. Aquatic Toxicology, 132-133, 61-72 (2013)

25. Jeng, H.A. and Swanson, J. Toxicity of Metal Oxide Nanoparticles in Mammalian Cells. J. Environ. Sci. Heal. A, 41, 2699-2711 (2006)

26. Oomen, A., Bos, P., Fernandes, T., Hund-Rinke, K., Boraschi, D., Byrne, H. J., Aschberger, K., Gottardo, S., von der Kammer, F., Kühnel, D., Hristozov, D., Marcomini, A ., Migliore, L., Scott-Fordsmand, J., Wick, P. and Landsiedel, R. Concern-driven integrated toxicity testing strategies for nanomaterials - Report of the NanoSafety Cluster Working Group 10. Nanotox., 8, 334-348 (2014)

27. Clark, K.A., White, R.H. and Silbergeld, E. K. Predictive models for nanotoxicology: Current challenges and future opportunities. Regul. Toxicol. Pharm., 59, 361-512 (2011)

28. European Union - Directive 2010/63/EU of the European Parliament and of the Council of 22 September 2010 on the protection of animals used for scientific purposes. (2010)

29. United States 106th Congress - Public Law 106-545, Dec. 19, 2000. 
30. Russell, W.M.S. and Burch, R.L. The Principles of Humane Experimental Technique.

London: Methuen (1959)

31. Organisation for Economic Co-Operation and Development (OECD): Guidance Document On Developing and Assessing Adverse Outcome Pathways, OECD, ENV/JM/MONO(2013)6, 2013.

32. Organisation for Economic Co-Operation and Development (OECD): Guidance Document on the Validation of (Quantative) Structure Activity Relationships [(Q)SAR] Models, OECD, ENV/JM/MONO(2007)2, 2007.

33. Puzyn, T., Leszczynska, D. and Leszczynski, J. Toward the Development of "Nano-QSARs": Advances and Challenges, Small, 22, 2494-2509 (2009)

34. Tomalia, D.A. and Fréchet, J.M.J. Discovery of Dendrimers and Dendritic Polymers: A Brief Historical Perspective. J. Polym. Sci. Pol. Chem., 40, 2719-2728 (2002)

35. Naha, P.C., Davoren, M., Casey, A. and Byrne H.J. An Ecotoxicological Study of Poly(amidoamine) Dendrimers-Toward Quantitative Structure Activity Relationships. Env. Sci. Technol., 43, 6864-6869 (2009)

36. Kitchens, K.M., Foraker, A.B., Kolhatkar, R.B., Swaan, P.W., Ghandehari, H. Endocytosis and interaction of poly (Amidoamine) dendrimers with Caco-2 cells. Pharmaceut. Res., 24, $2138-2145(2007)$

37. Hong, S., Bielinska, A.U., Mecke, A., Keszler, B., Beals, J.L., Shi, X., Balogh, L., Orr, B.G., Baker, J.R.Jr and Banaszak Holl, M.M. Interaction of poly(amidoamine) dendrimers with 
supported lipid bilayers and cells: hole formation and the relation to transport. Bioconjugate Chem., 15, 774-782 (2004)

38. Mukherjee, S.P. and Byrne H.J. Polyamidoamine dendrimer nanoparticle cytotoxicity oxidative stress, caspase activation and inflammatory response: experimental observation and numerical simulation. Nanomed: Nanotech. Bio. Med., 9, 202-211 (2013)

39. Guillot-Nieckowski, M., Eisler, S. and Diederich, F. Dendritic vectors for gene transfection. New J. Chem., 31, 1111-1127 (2000)

40. Kukowska-Latallo, J.F., Bielinska, A.U., Johnson, J., Spindler, R., Tomalia, D.A. and Baker, J.R.Jr. Efficient transfer of genetic material into mammalian cells using Starburst polyamidoamine dendrimers. P. Natl. Acad. Sci. USA, 93, 4897-4902 (1996)

41. Zhou, J., Wu, J., Hafdi, N., Behr, J.P., Erbacher, P. and Peng, L. PAMAM dendrimers for efficient siRNA delivery and potent gene silencing. Chem. Commun., 22, 2362-2364 (2006) 42. Atkinson, K.A. An Introduction to Numerical Analysis (2nd ed.), New York: John Wiley \& Sons, ISBN 978-0-471-50023-0 (1989)

43. Einstein, A. Strahlungs-emission und -absorption nach der Quantentheorie. Verhandlungen der Deutschen Physikalischen Gesellschaft, 18, 318-323 (1916)

44. Snow E.T., Sykora P., Durham T.R., Klein C.B. Arsenic, mode of action at biologically plausible low doses: What are the implications for low dose cancer risk? Toxicol. Appl. Pharm., 207, S557 - S564 (2005) 
45. Nakagawa, Y., Akao, Y., Morikawa, H., Hirata, I., Katsu, K., Naoe, T., Ohishi, N. and Yagi, K. Arsenic trioxide-induced apoptosis through oxidative stress in cells of colon cancer cell lines. Life Sciences, 70, 2253-2269 (2002)

46. Shen, L. and Sevanian, A. OxLDL induces macrophage $\gamma$-GCS-HS protein expression: a role for oxLDL-associated lipid hydroperoxide in GSH synthesis. J. Lipid Res., 42, 813-823 (2001)

47. Nociari, M.M., Shalev, A., Benias, P. and Russo, C. A novel one-step, highly sensitive fluorometric assay to evaluate cell-mediated cytotoxicity. J. Immunol. Methods, 213, 157-167 (1998)

48. Repetto, G., del Peso, A. and Zurita, J.L. Neutral red uptake assay for the estimation of cell viability/cytotoxicity. Nature Protocols, 3, 1125-1131 (2008)

49. Ankley, G.T., Bennett, R.S., Erickson, R.J., Hoff, D.J., Hornung, M.W., Johnson, R.D., Mount, D.R., Nichols, J.W., Russom, C.L., Schmieder, P.K., Serrrano, J.A., Tietge, J.E. and Villeneuve, D.L. Adverse Outcome Pathways: A conceptual framework to support ecotoxicology research and risk assessment. Environ. Toxicol. Chem., 29, 730-741 (2010)

50. Meng, T., Xia, T., George, S., and Nel, A.E. A Predictive Toxicological Paradigm for the Safety Assessment of Nanomaterials, ACS Nano, 3, 1620 -1627 (2009)

51. Crooks, R.M., Lemon III, B.I., Yeung, L.K. and Zhao, M. Dendrimers III: Design, Dimension, Function: Dendrimer-encapsulated Metals and Semiconductors: Synthesis, Characterisation and Applications, Topics in Current Chemistry, 212, 81-135 (2001)

52. Black, J.W. and Leff, P. Operational models of pharmacological agonism. Proceeding of the Royal Society B: Biological Sciences, 220, 141-162 (1983) 
53. Issels, R.D., Nagele, A., Eckert, K-G. and Wilmanns W. Promotion of cystine uptake and its utilization for glutathione biosynthesis induced by cysteamine and $\mathrm{N}$-acetylcysteine. Biochem. Pharmacol., 37, 1029-1033 (1985)

54. Tsan, M.F., Danis, E.H., Del Vecchio, P.J. and Rosano, C.L. Enhancement of intracellular glutathione protects endothelial cells against oxidant damage. Biochem. Bioph. Res. Co., 127, 270-276 (1985)

55. Tsan, M.F., White, J.E. and Rosano, C.L. Modulation of endothelial GSH concentrations: effect of exogenous GSH and GSH monoethyl ester. J. Appl. Physiol., 66, 1029-1034 (1989)

56. Vlahopoulos, S., Boldogh, I., Casola, A. and Brasier, A.R. Nuclear Factor-kB-Dependent Induction of Interleukin-8 Gene Expression by Tumor Necrosis Factor- $\alpha$ : Evidence for an Antioxidant Sensitive Activating Pathway Distinct From Nuclear Translocation. Blood., 94, 1878-1889 (1999)

57. Deneke, S.M. and Fanburg, B.L. Regulation of cellular glutathione. Am. J. Physiol., 257, L163-L173 (1989)

58. Miyazaki, M., Nakade, S., Iwanaga, K., Morimoto, K., and Kakemi, M. Estimation of bioavailability of salmon calcitonin from the hypocalcemic effect in rats (I): pharmacokineticpharmacodynamic modeling based on the endogenous Ca regulatory system. Drug Metabol. Pharmacokinetics, 18, 350-357 (2003)

59. Ryan, S.M., Frías, J.M., Wang, X., Sayers. C.T., Haddleton, D.M. and Brayden, D.J. PK/PD modelling of comb-shaped PEGylated salmon calcitonin conjugates of differing molecular weights. J. Controlled Release, 149, 126-132 (2011) 
60. Dell'Orco, D., Lundqvist, M., Cedervall, T. and Linse, S. Delivery success rate of engineered nanoparticles in the presence of the protein corona: a systems-level screening. Nanomed: Nanotech. Bio. Med., 8, 1271-1281 (2012)

61. Zi, Z., Zheng, Y., Rundell, A.E. and Klipp, E. SBML-SAT: a systems biology markup language (SBML) based sensitivity analysis tool. BMC Bioinformatics, 9, 342 (2008)

62. Bennett, E.R., Clausen, J., Linkov, E. and Linkov, I. Predicting physical properties of emerging compounds with limited physical and chemical data: QSAR model uncertainty and applicability to military munitions. Chemosphere, 77, 1412-1418 (2009) 


\section{$\underline{\text { Numerical simulations of in vitro nanoparticle toxicity - Towards }}$}

\section{structure activity relationships and predictive models for toxicology}

\section{and medicine.}

Marcus A. Maher", Pratap C. Naha ${ }^{\dagger}$, Sourav Prassana Mukerjee ${ }^{\S}$, Hugh J. Byrne

Focas Research Institute, Dublin Institute of Technology, Kevin Street, Dublin 8, Ireland.

$\dagger$ Now at: Department of Radiology, Perelman School of Medicine, University of Pennsylvania, Philadelphia, Pennsylvania 19104, USA

$\S$ Now at: Health Canada, Mechanistic Studies Division, Ottawa, Ontario, Canada

\section{Supplementary Information:}

Table S1: Parameters employed for the final simulated datasets.

\begin{tabular}{|l|l|}
\hline$d t$ & $0.1 \mathrm{hr}$ \\
\hline$k_{A}$ & $0.0011 \mathrm{hr}^{-1}$ \\
\hline$R T_{0}$ & $10 \mu \mathrm{M}$ \\
\hline$k_{\text {endo }}$ & $0.75 \mathrm{hr}^{-1}$ \\
\hline$c$ & 0.25 \\
\hline$k_{G S H}$ & $1.00 \mathrm{hr}^{-1}$ \\
\hline$k_{q}$ & $0.40 \mathrm{hr}^{-1}$ \\
\hline$k_{\lambda}$ & $(\ln 2 / 24) \mathrm{hr}^{-1}$ \\
\hline$B$ & 3 \\
\hline
\end{tabular}




\begin{tabular}{|l|l|}
\hline$N_{\text {source }}(t=0)$ & $10 \mu \mathrm{M}$ \\
\hline$k_{\text {casp }}$ & $0.02 \mathrm{hr}^{-1}$ \\
\hline$k_{\text {casp } 2}$ & $0.30 \mathrm{hr}^{-1}$ \\
\hline$k_{m m p}$ & $0.001 \mathrm{hr}^{-1}$ \\
\hline$k_{\text {trf }}$ & $0.035 \mathrm{hr}^{-1}$ \\
\hline$k_{\text {il }}$ & $0.10 \mathrm{hr}^{-1}$ \\
\hline$k_{V}$ & $8.00 \mathrm{hr}^{-1}$ \\
\hline$k_{\text {rec }}$ & $0.065 \mathrm{hr}^{-1}$ \\
\hline$k_{R O S}$ & $1.20 \mathrm{hr}^{-1}$ \\
\hline
\end{tabular}

In previous work ${ }^{\mathrm{SR} 1}$, the time dependent rise and subsequent decay of the generation of ROS was modelled according to equation (10). However, such a model does not accurately account for the observed dose dependence, as shown in figure S1. Although an initial saturation-like behaviour is observable at intermediate doses, at higher doses the levels of ROS continue to increase monotonically.

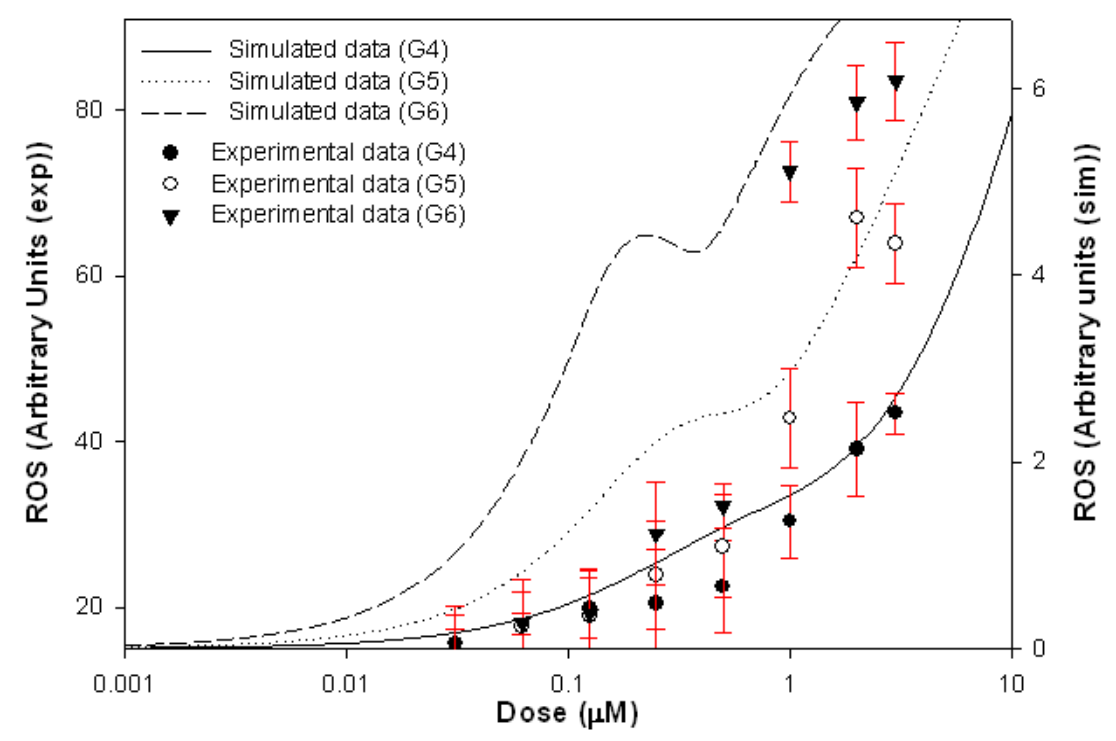


Figure S1: Dose dependent ROS generation for all generations simulated according to Equation 10.

The experimentally observed dose and time dependence of the increased levels of ROS is faithfully reproduced, however, by invoking the concept of a source of ROS which is depleted according to Equation 11, resulting in a dose and time dependent generation of ROS according to Equation 12 as shown in Figure 2.

Figure $\mathrm{S} 2$ shows the simulated uptake of nanoparticles, $\mathrm{N}_{\text {endo }}$, calculated according to Equation 2, for PAMAM generations G4, G5, G6, with values of $c=0.25, k_{\square \square}=(\ln 2 / 24) h r^{-1}$. Similar to the behaviour observed by Salvati et al. ${ }^{\mathrm{SR} 2}$ for aminated polystyrene nanoparticles, the response increases approximately linear at early times, but deviates from linearity as the cells divide and the nanoparticles are distributed over a larger cell population. Although no values of $k_{\text {endo }}$ are quoted by Salvati et al., the experimental data in their study are best fitted by a regime in which $k_{\text {endo }}>k_{\square}$. In figure S1, $k_{\text {endo }}=0.75 \mathrm{hr}^{-1}>k_{\square}=0.03 \mathrm{hr}^{-1}$. 


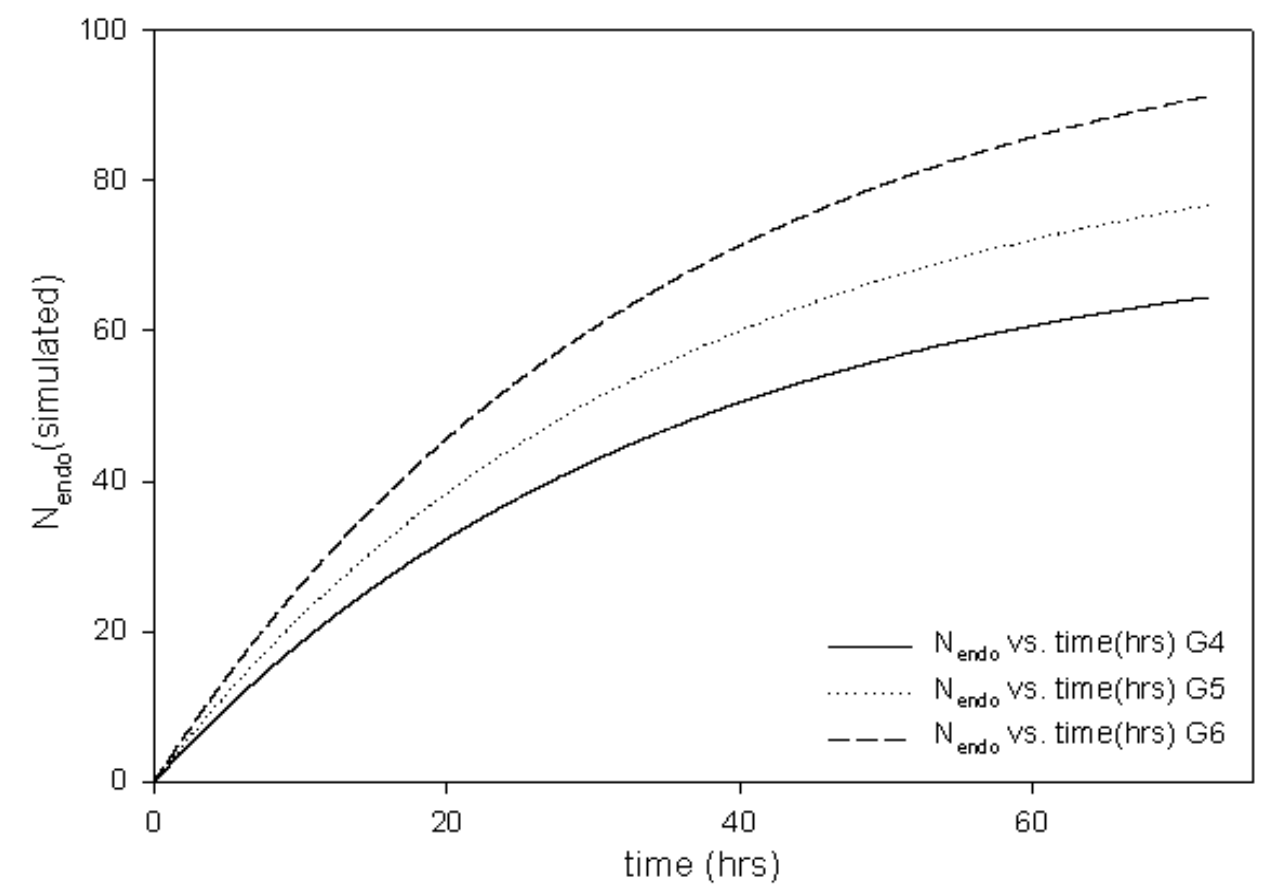

Figure S2: Simulated time dependent uptake of PAMAM dendrimers of generation G4, G5, G6, according to Equation 2, at a fixed dose of $1 \square \mathrm{m}$.

The effect of variation in $k_{\text {endo }}$ is also manifest in the dose dependent evolution of the ROS levels for all generations, as shown in Figure S3, for the example of $4 \mathrm{hrs}$ exposure. Variation of $k_{\text {endo }}$ has the effect of systematically shifting the median dose point of ROS increase. The best reproduction of the experimentally observed behaviour for the combined dataset of three dendrimer generations was found with $k_{\text {endo }}=0.75 \mathrm{hr}^{-1}$.

Confocal microscopic studies of mammalian cell exposure to PAMAM dendrimers by Mukherjee et al. have clearly demonstrated co-localisation of the early stage ROS with endosomes. ${ }^{\text {SR3,SR4 }}$ In the case of cationic nanoparticles in the acidifying environment of endosomes, it is reported that the unsaturated surface amino groups sequester protons that are supplied by the v-ATPase 
(proton pump). ${ }^{\text {SR5 }}$ This process keeps the pump functioning and leads to the retention of one $\mathrm{Cl}^{-}$ ion and one water molecule per proton. It has been proposed that the initial wave of ROS may be produced via NADPH oxidase. ${ }^{\text {SR6 }}$ NADPH oxidase is an enzyme which produces superoxide anions $\left(\mathrm{O}_{2}^{-}\right)$in phagosomes and endosomes. ${ }^{\mathrm{SR} 7}$ Evidence also points to the release of the superoxide to the cytoplasm via chloride anion channels. ${ }^{\text {SR7 }}$ Superoxide production is accomplished by an electron transfer from NADPH, via FAD, to molecular oxygen $\left(\mathrm{O}_{2}\right)$. The $\mathrm{O}_{2}^{-}$ then rapidly dismutases to hydrogen peroxide $\left(\mathrm{H}_{2} \mathrm{O}_{2}\right) .{ }^{\mathrm{SR} 8}$ It is also believed that the protons from the proton pump mechanism are used in the dismutation of superoxide. NADPH oxidase is present in nearly all cell types (including $\mathrm{HaCaT}$ cells ${ }^{\mathrm{SR} 9}$ ) and is important in both host defences and redox signalling. Reviewed by SR10 and SR11 NADPH oxidase has been implicated in the production of ROS in nanoparticle induced toxicity for other nanoparticles, including silica, etc. ${ }^{\text {SR12,SR13 }}$ The idea that NADPH oxidase is responsible for PAMAM induced toxicity deserves further attention and may aid in a deeper understanding of the underlying processes involved in nano-cytotoxicity. 
A:

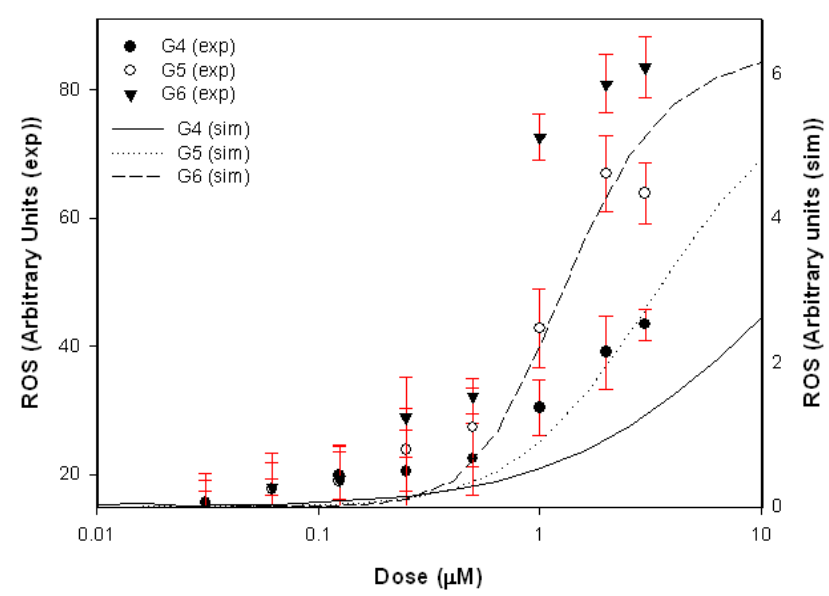

Figure $\quad$ S3-A: $\quad k_{\text {endo }}=0.25 h r^{-1}$

B:

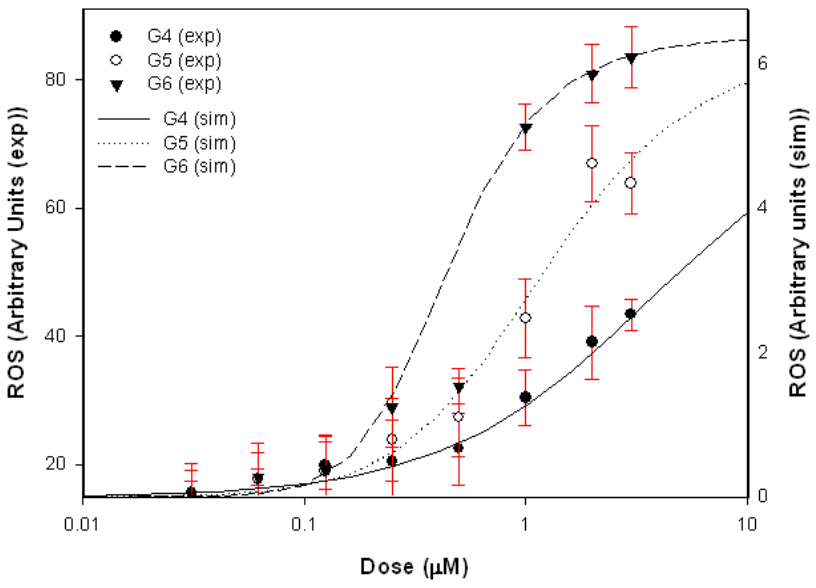

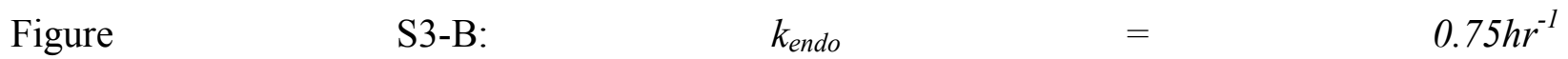


$\mathrm{C}:$

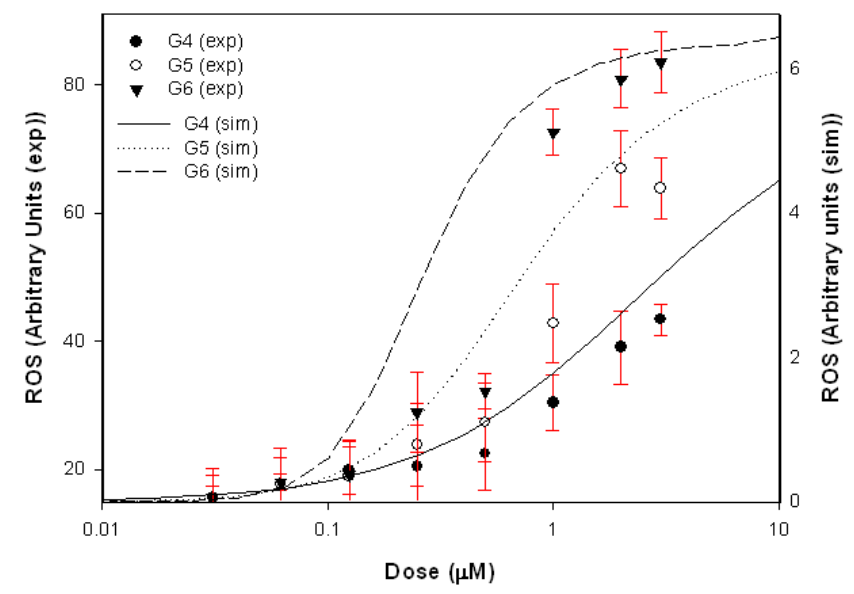

Figure S3-C: $k_{\text {endo }}=1.25 \mathrm{hr}^{-1}$

Figure S3: Variation in simulated (lines) increase in ROS levels on the parameter $k_{\text {endo }}$ in Equation 2, compared to experimental data (symbols) for G4, G5, G6. A: $k_{\text {endo }}=0.25 h r^{-1}$, B: $k_{\text {endo }}=0.75 \mathrm{hr}^{-1}, \mathrm{C}: k_{\text {endo }}=1.25 \mathrm{hr} \mathrm{r}^{-1}$.

The generation dependence of the uptake (also demonstrated in Mukherjee, S.P. and Byrne H.J. $2013^{\mathrm{SR} 1}$ ) derives from the term $\left(N_{a m g}\right)^{c}$ in Equation 2, and although the uptake of the dendrimers cannot be directly visualised, the effect of this term on the generation dependent increase in ROS levels is demonstrated in figure S4. The generation dependent factor similarly has the effect of shifting the dose dependent curves along the dose axis, but also systematically varies the separation of the responses for the different dendrimer generations. The best reproduction of the ROS curves was obtained using a value of $\mathbf{c}=0.25$, as shown in figure S4:B. 
A:

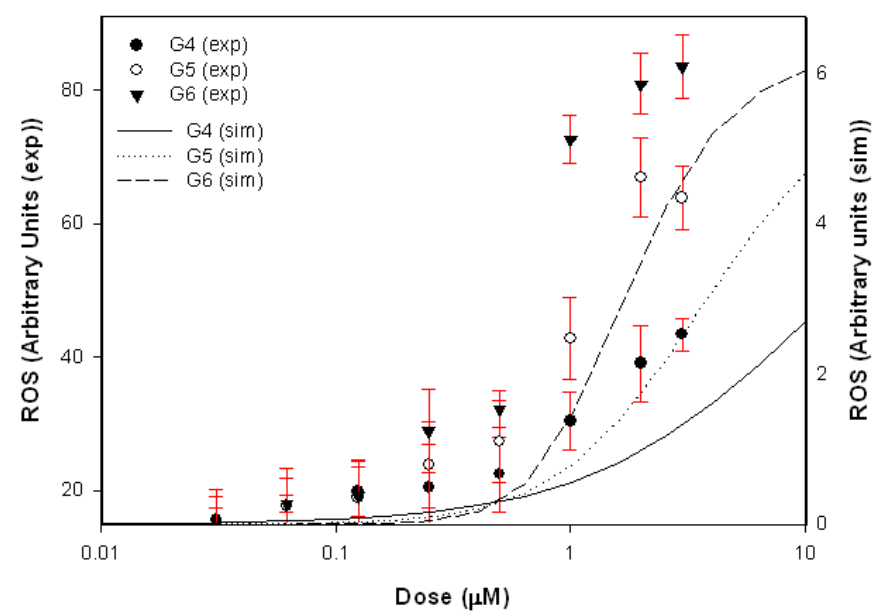

Figure

S4-A:

$c$

B:

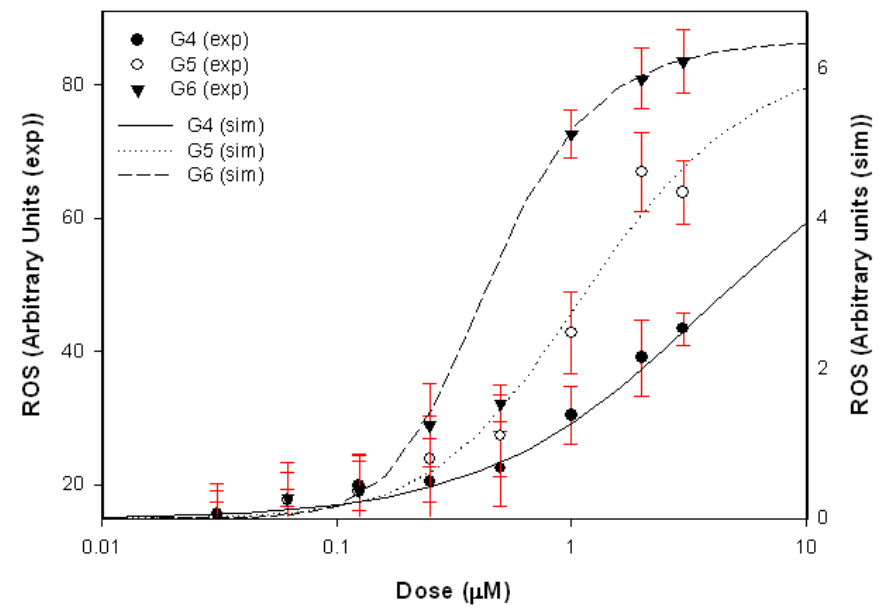

Figure S4-B: $c=0.25$ 
C:

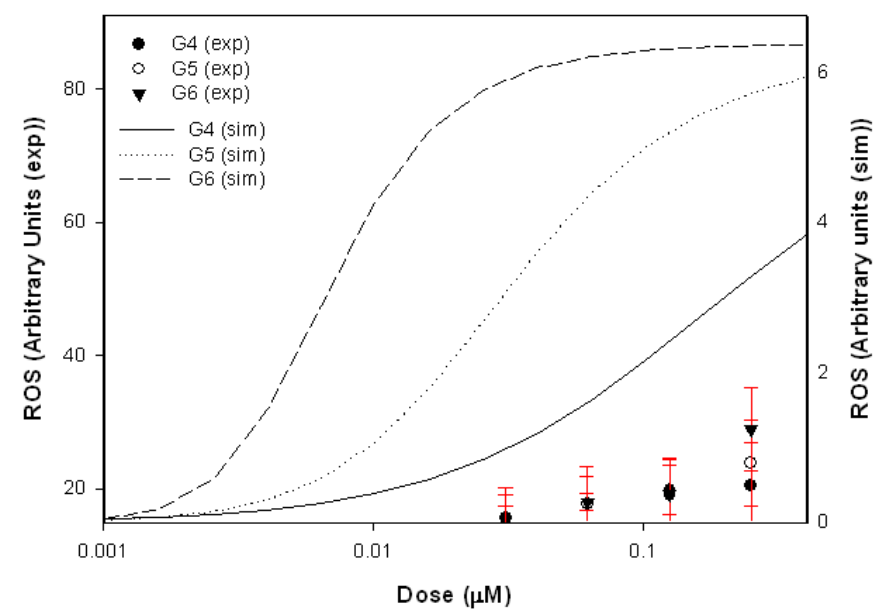

Figure S4-C: $c=1$

Figure S4: Variation in simulated (lines) increase in ROS levels on the parameter $\left(N_{\text {amg }}\right)^{c}$ in Equation 2, compared to experimental data (symbols) for G4, G5, G6. A: $c=0, \mathrm{~B}: c=0.25$ and $\mathrm{C}: c=1$.

Figure S5 illustrates the sensitivity of the simulations to variations in the parameter $k_{A}$ in Equations 11 and 12. The parameter largely impacts on the separation of the dose dependences of the different generations. The best reproduction of the experimental behaviour was observed with a value of $k_{A}=0.0011 \mathrm{hr}^{-1}$ 
A:

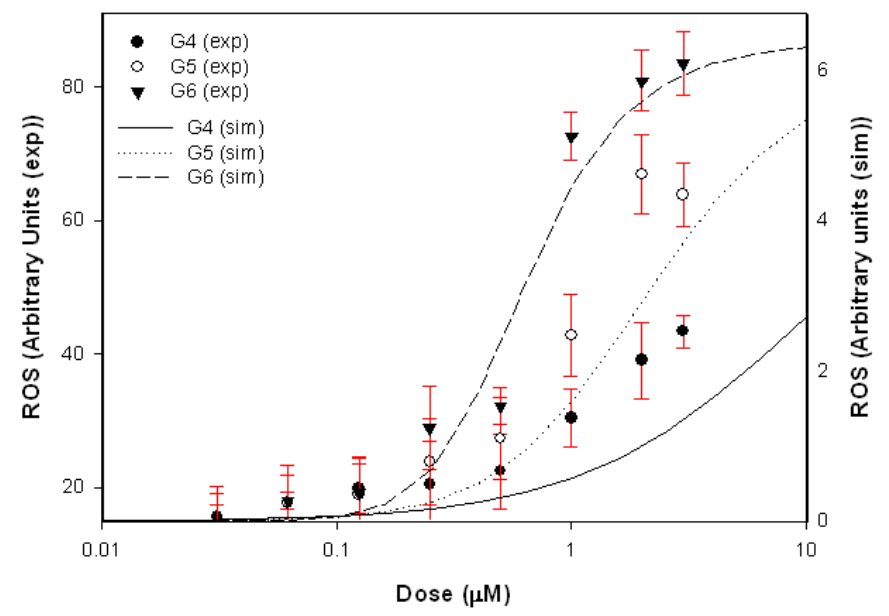

$\begin{array}{lllll}\text { Figure } & \text { S5-A: } & k_{A} & = & 0.0004\end{array}$

B:

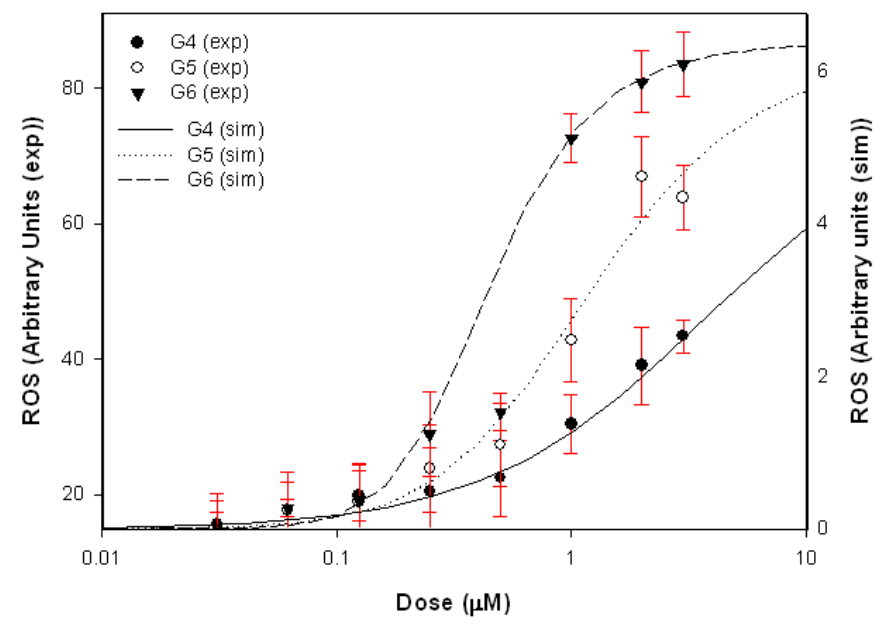

$\begin{array}{lllll}\text { Figure } & \text { S5-B: } & k_{A} & = & 0.0011\end{array}$ 
C:

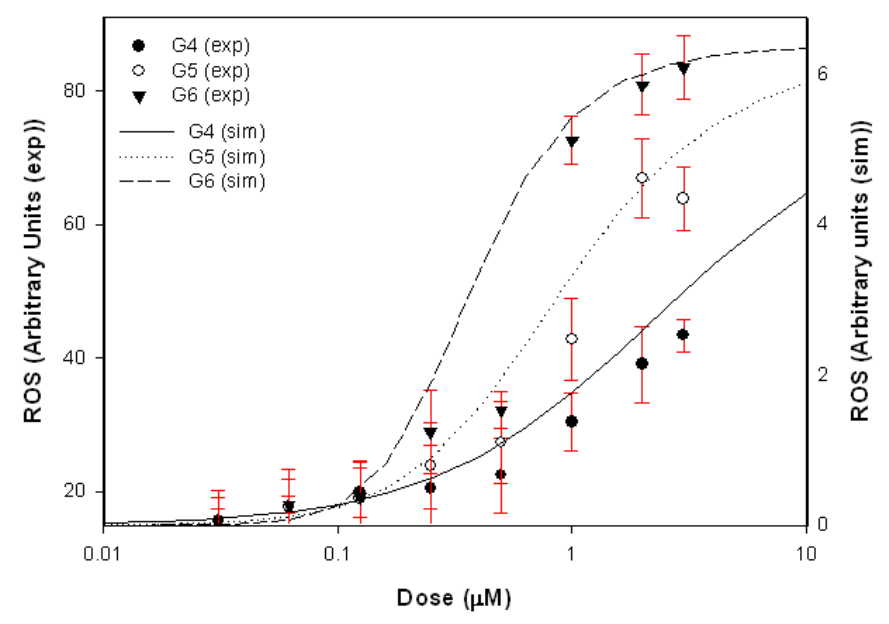

$\begin{array}{lllll}\text { Figure } & \text { S5-C: } & k_{A} & = & 0.0018\end{array}$

Figure S5: Variation in simulated (lines) increase in ROS levels on the parameter $k_{A}$ compared to experimental data (symbols) for G4, G5, G6. A: $k_{A}=0.0004 h r^{-1}$, B: $k_{A}=0.0011 h r^{-1}$ and C: $k_{A}=$ $0.0018 \mathrm{hr}^{-1}$.

Figure S6 shows how the dose dependant ROS values vary with the $G^{*}$ parameter. Since $G^{*}=$ $1 /\left(N_{\text {amg }}{ }^{0.25}\right)$ the variance was observed by changing the exponent value $(0.25)$. 
A:

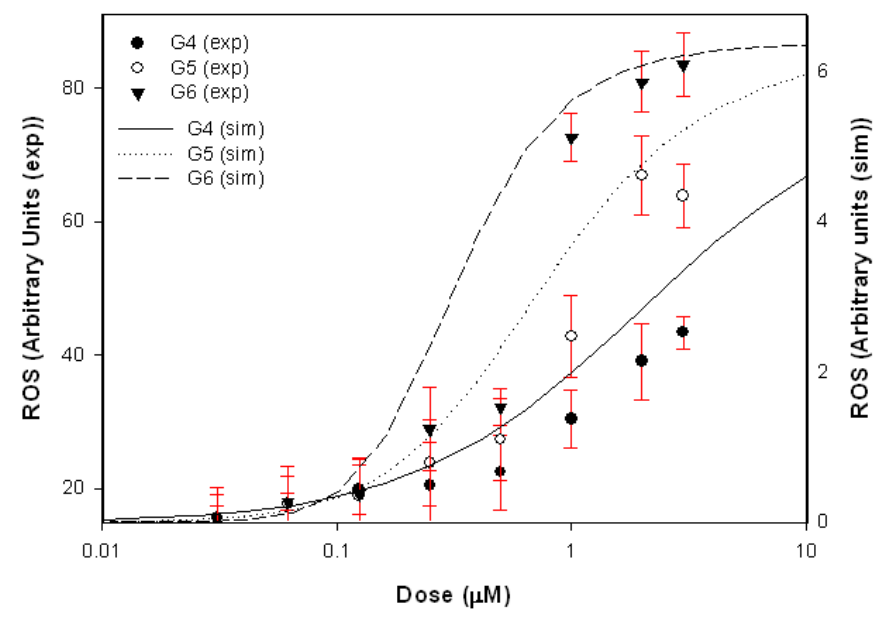

$\begin{array}{llll}\text { Figure S6-A: } & \text { Exponent } & \text { value } & 0.083\end{array}$

B:

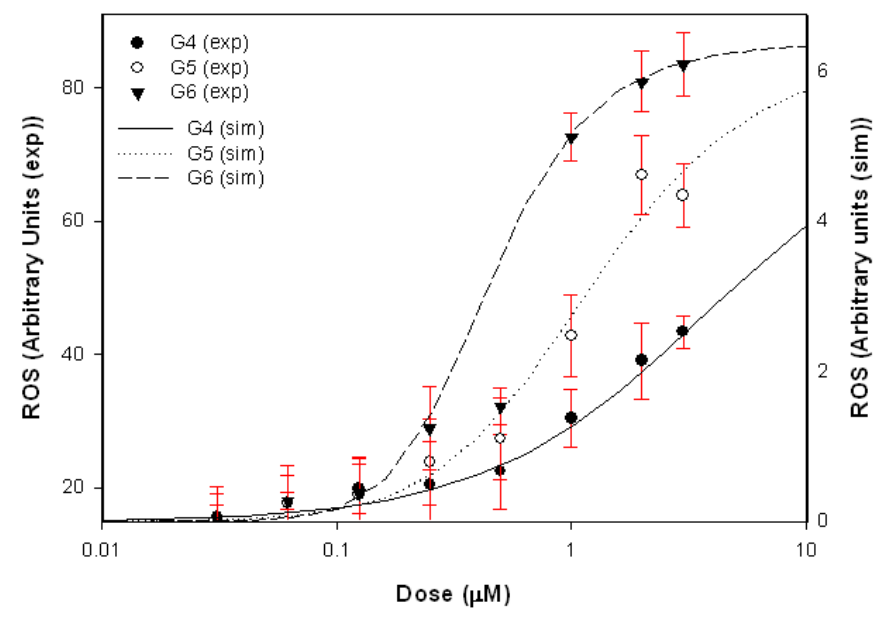

$\begin{array}{lllll}\text { Figure } & \text { S6-B: } & \text { Exponent } & & 0.25\end{array}$

C: 


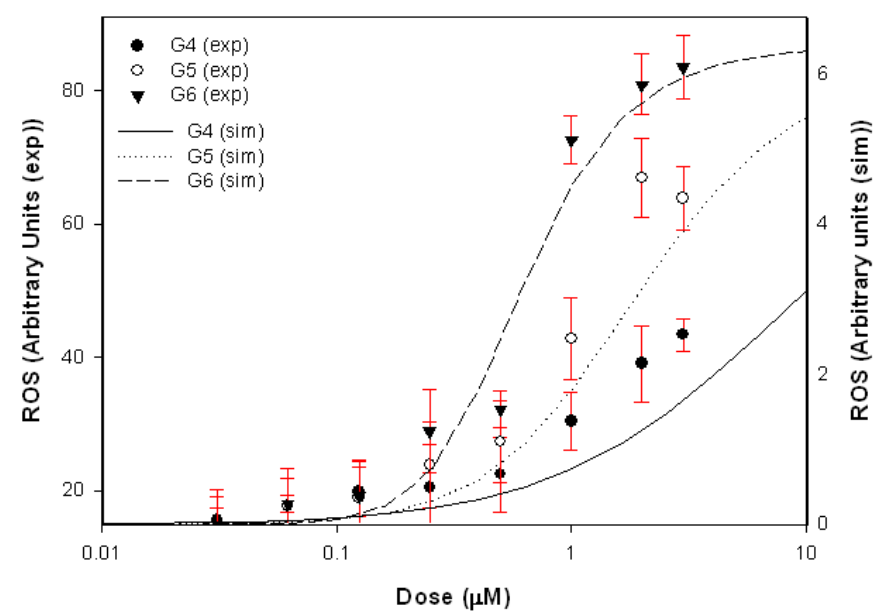

Figure S6-C: Exponent value $=0.42$

Figure S6(A-C): Variation in simulated (lines) increase in ROS levels on the exponential value in the equation $G^{*}=1 /\left(N_{a m g}{ }^{0.25}\right)$, compared to experimental data (symbols) for G4, G5, G6. A: Exponent value $=0.083$, B: Exponent Value $=0.25, \mathrm{C}:$ Exponent Value $=0.42$.

Figure S7 shows how the dose dependant ROS values vary with the parameter A. Since $A=$ $\left(N_{a m g} / 64\right)^{0.75}$ the variance was observed by changing the exponent value $(0.75)$.

A:

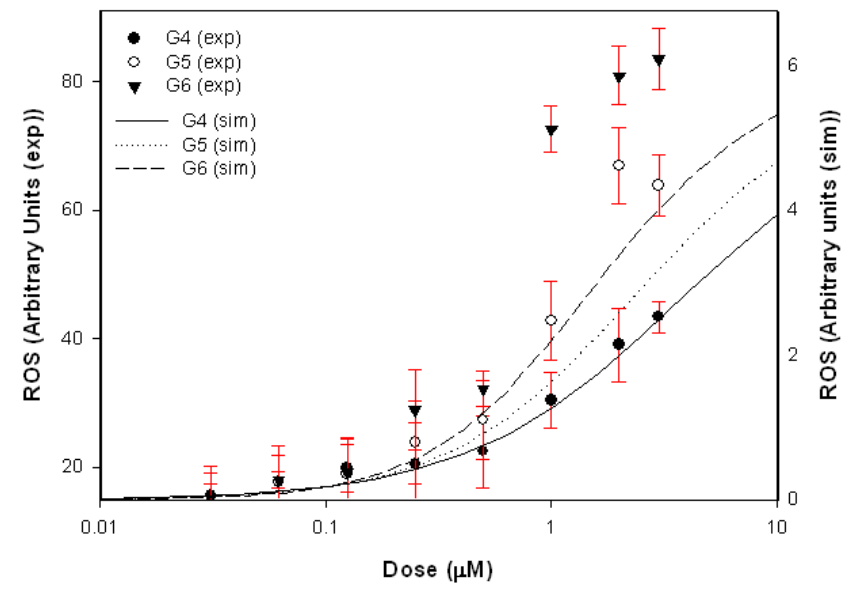

$\begin{array}{llll}\text { Figure } & \text { S7-A: } & \text { value } & \end{array}$

B: 


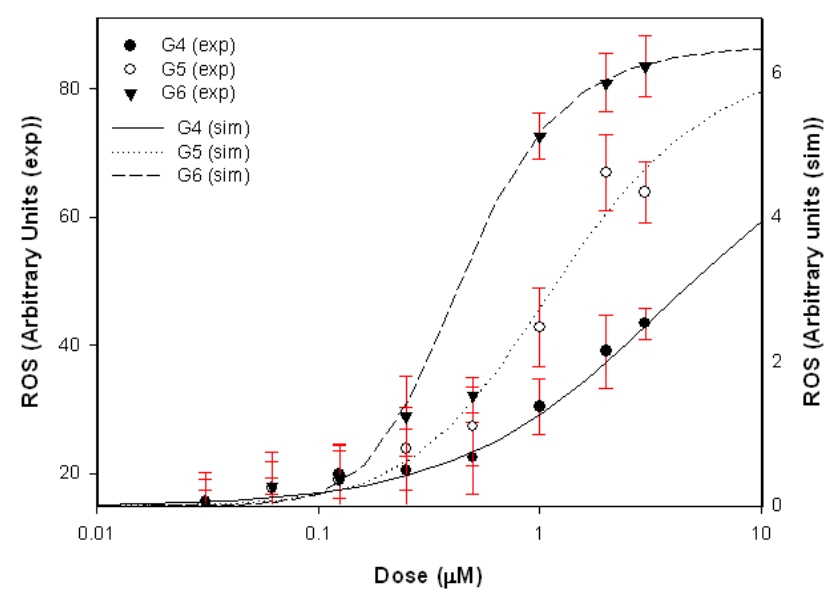

Figure

S7-B:

Exponent

value

$=$

0.75

C:

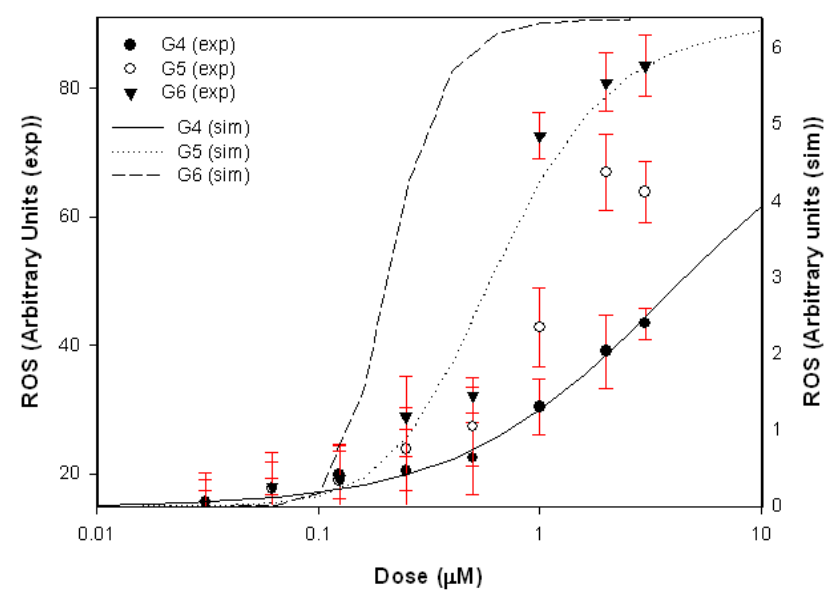

Figure S7-C: Exponent value $=1.25$

Figure S7(A-C): Variation in simulated (lines) increase in ROS levels on the exponential value in the equation $A=\left(N_{a m g} / 64\right)^{0.75}$, compared to experimental data (symbols) for G4, G5, G6. A: Exponent value $=0.25, \mathrm{~B}:$ Exponent Value $=0.75, \mathrm{C}:$ Exponent Value $=1.25$.

Figure S8(A-C) shows how the does dependant ROS production varies with the parameter B of Equations 11 and 12. It can be seen that this parameter has a dramatic effect on the overall shape, dose and generation dependence of the curves. A best reproduction of the experimental behaviour was obtained with $B=3$. 
A:

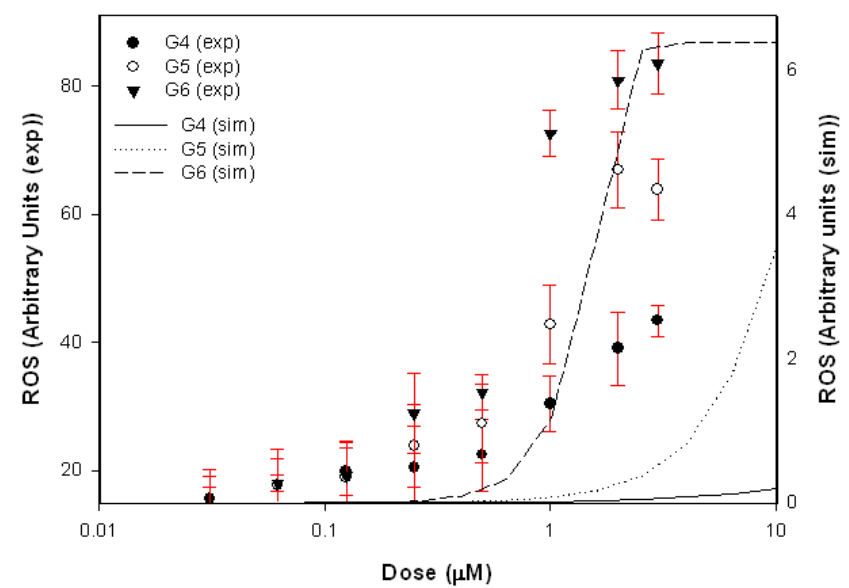

Figure

S8-A:

B

B: 


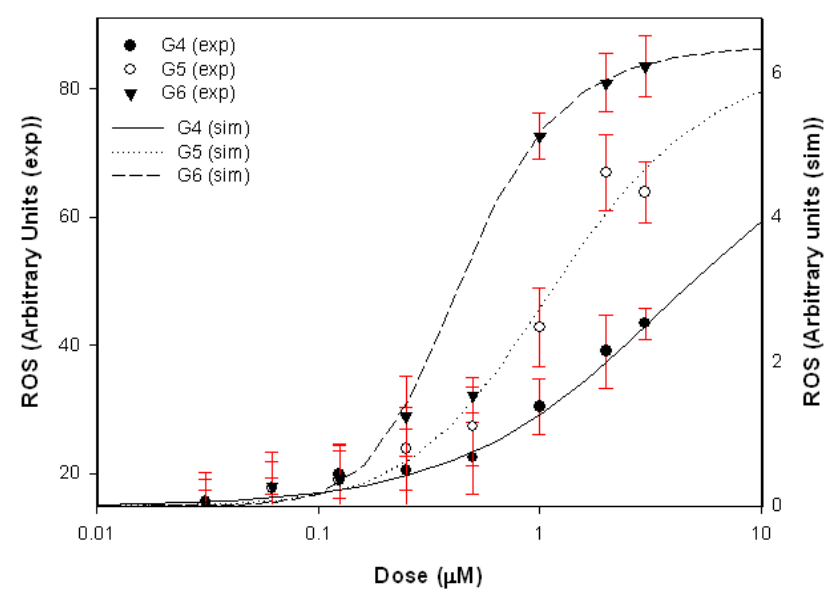

Figure

S8-B:

$B$

C:

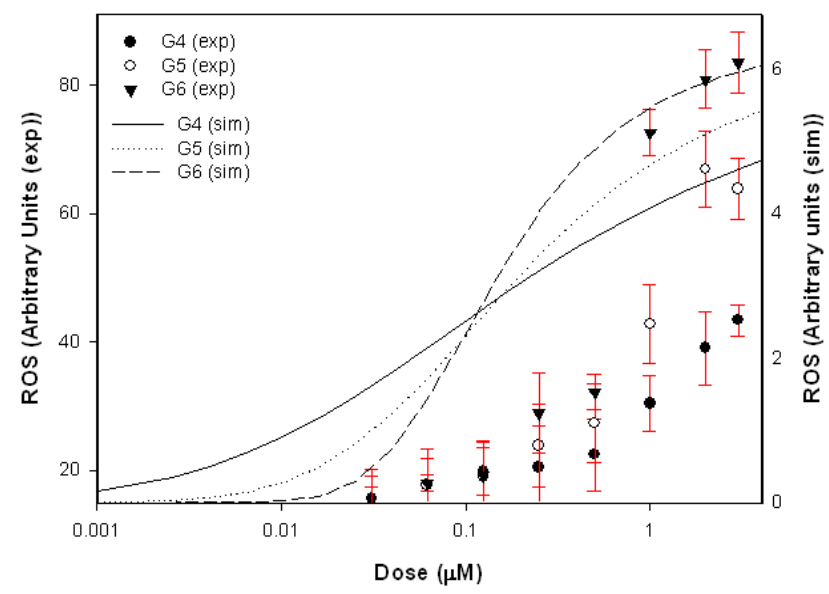

Figure

S8-C:

$B$

Figure S8: Variation in simulated (lines) increase in ROS levels on the parameter $B$ in Equation 11/12, compared to experimental data (symbols) for G4, G5, G6. A: $B=1, \mathrm{~B}: B=3$ and $\mathrm{C}: B=$ 5.

Figure S9 shows how the viability of the cells (for G4 at three timepoints), as calculated using Equation (9) is affected by the parameter $k_{M M P}$. 
A:

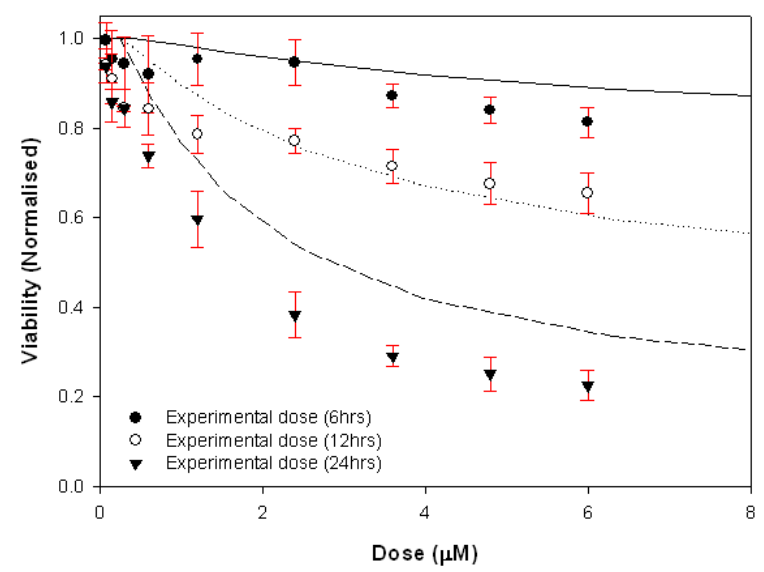

Figure

S9-A:

$k_{M M P}$

$=$

0.0007

B:

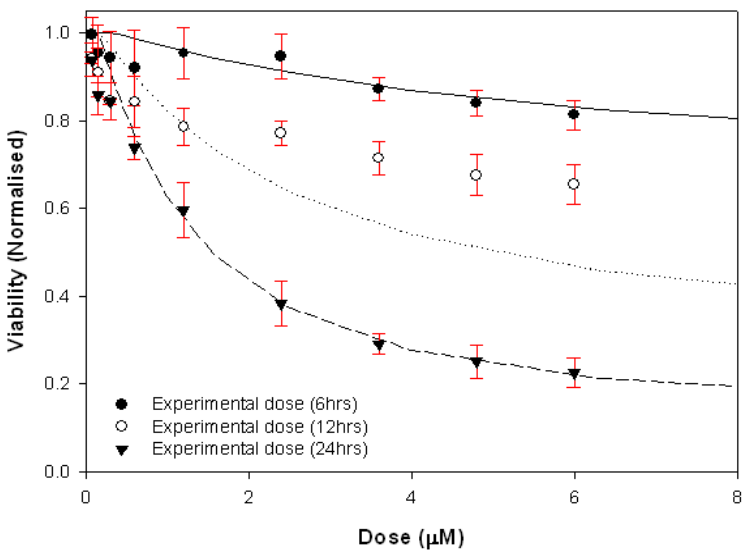

Figure

S9-B:

$k_{M M P}$

$=$

0.0010

C: 


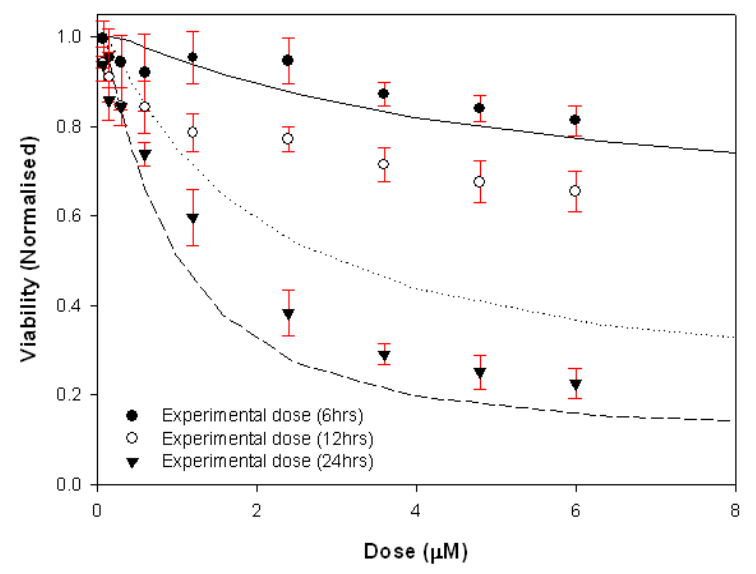

Figure S9-C: $k_{M M P}=0.0013$

Figure S9(A-C): Effect of variation of the parameter $k_{M M P}$ on simulated (lines) viability compared to experimental data (symbols) for G4 at timepoints: 6hrs, $12 \mathrm{hrs}$ and $24 \mathrm{hrs}$. A: $k_{M M P}=$ $0.0007, \mathrm{~B}: k_{M M P}=0.0010$ and $\mathrm{C}: k_{M M P}=0.0013$.

Figure S10 shows how the viability of the cells (for 3 generations of nanoparticle), as calculated using Equation (9) is affected by the parameter $k_{M M P}$.

A:

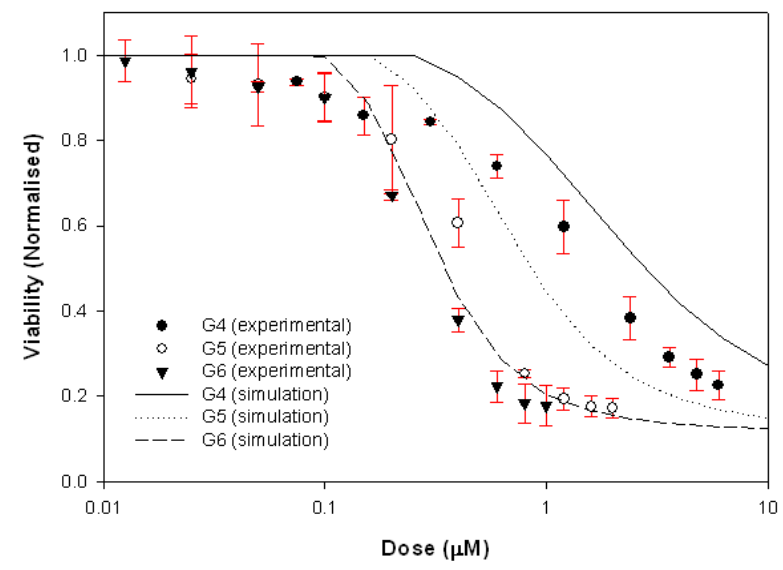


Figure

B:

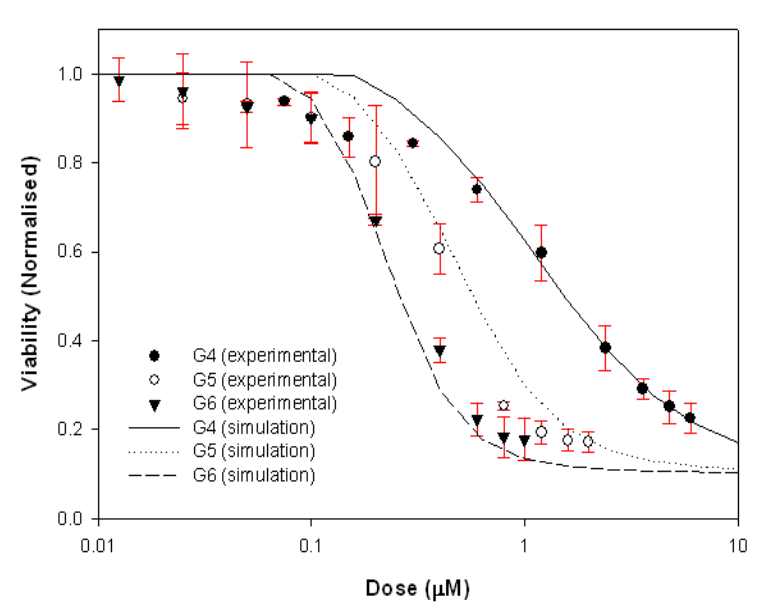

C:

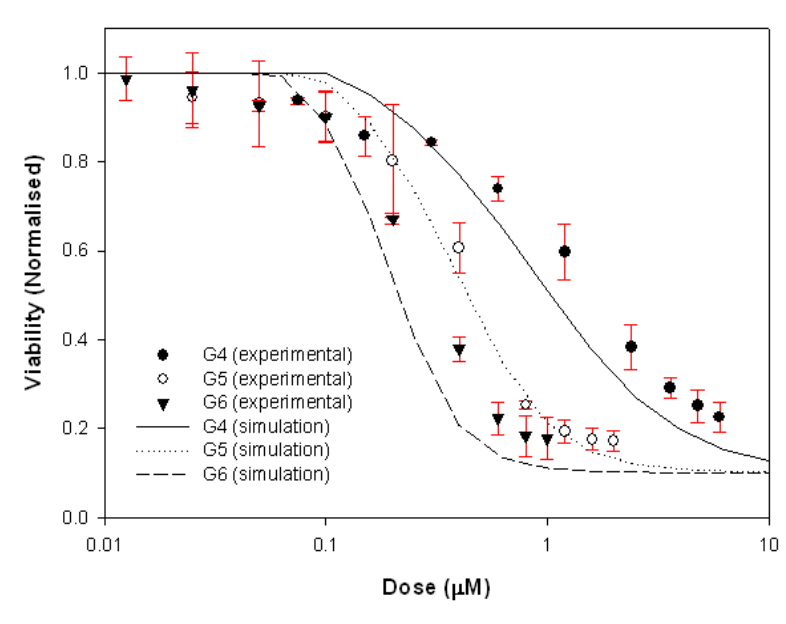

Figure S10-C: $k_{M M P}=0.0013$

S10-A:

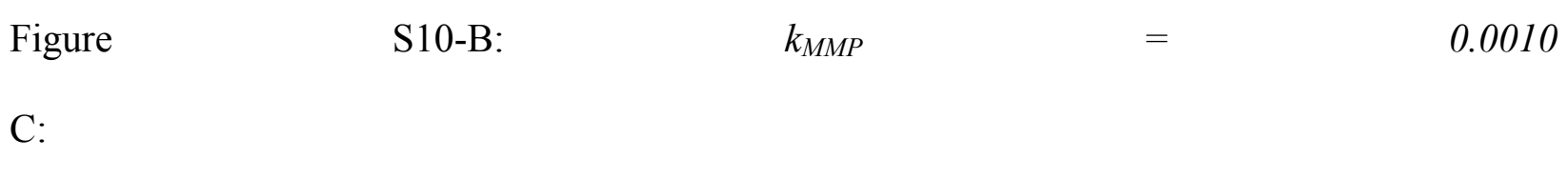

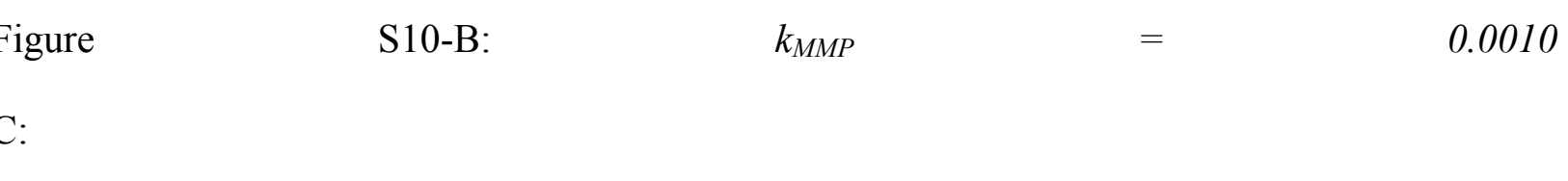

$k_{M M P}$

$=$

0.0007

.

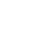

0.0007

Figure S10(A-C): Effect of variation of the parameter $k_{M M P}$ on simulated (lines) viability compared to experimental data (symbols) for G4, G5, G6. A: $k_{M M P}=0.0007$, B: $k_{M M P}=0.0010$ and $\mathrm{C}: k_{M M P}=0.0013$. 
Figure S11 shows how the viability of the cells, as calculated using Equation (9) is affected by the exponent value " $m$ " in equation 13 .

A:

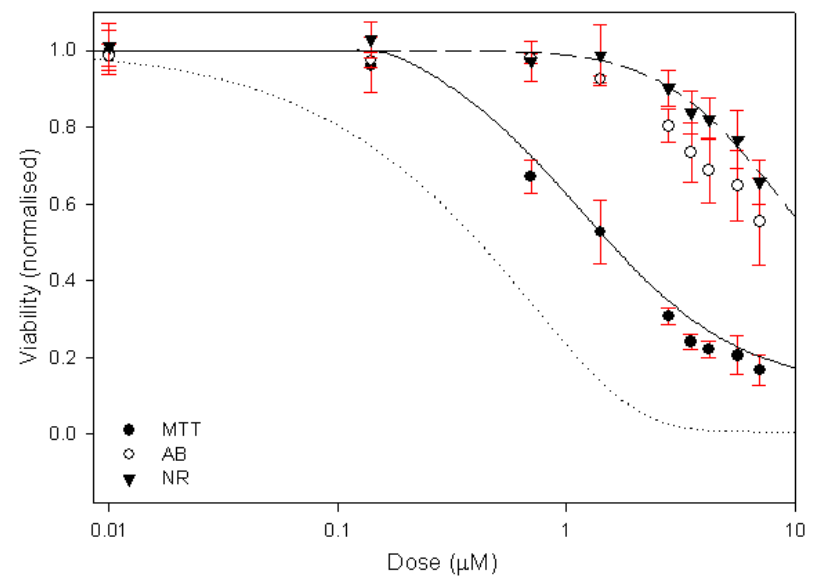

Figure 11-A: $m=1$

B:

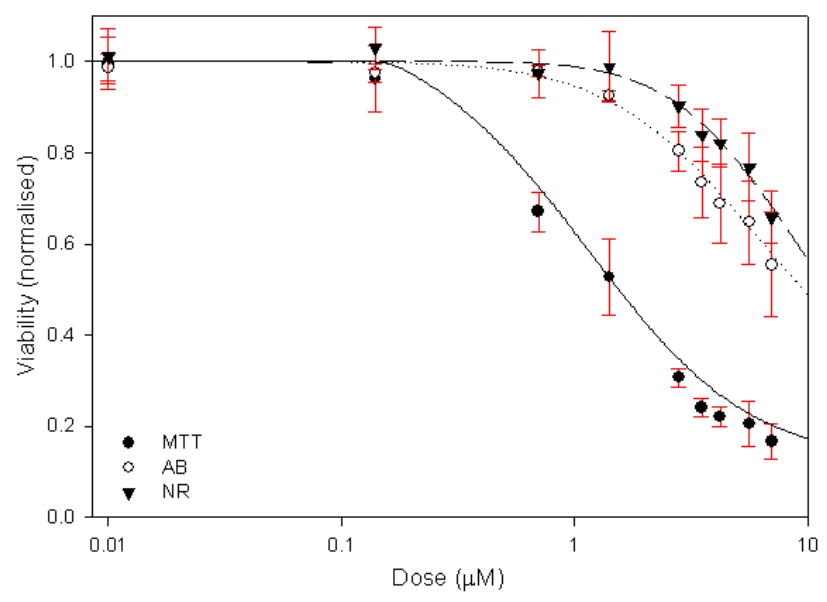

Figure 11-B: $m=2$

C: 


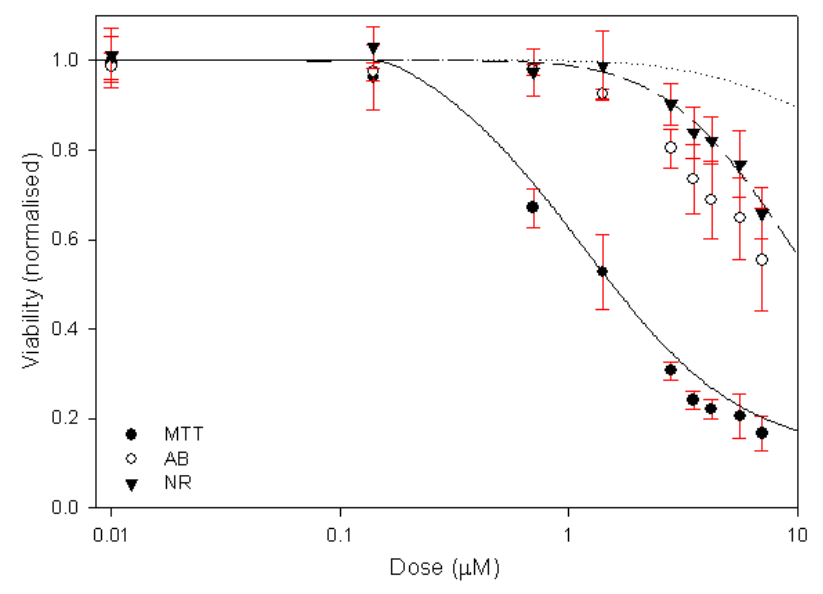

Figure 11-C: $m=3$

Figure S11(A-C): Effect of variation of the parameter $m$ (dotted line) on simulated (lines) viability compared to experimental data (symbols) for 3 different assay types . A: $m=1, \mathrm{~B}: m=$ 2 and $\mathrm{C}: m=3$.

Figure S12 shows how the viability of the cells, as calculated using Equation (9) is affected by the exponent value " $p$ " in equation 14 .

A:

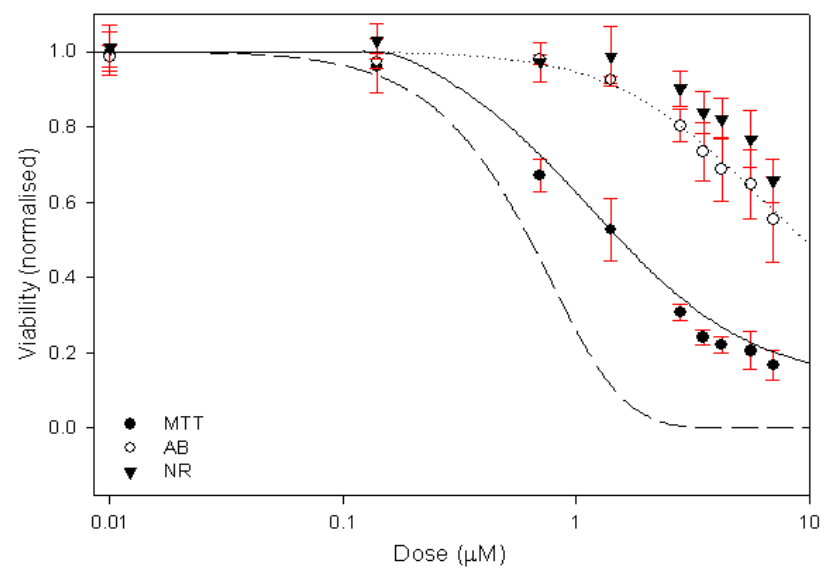

Figure 12-A: $p=2$ 
B:

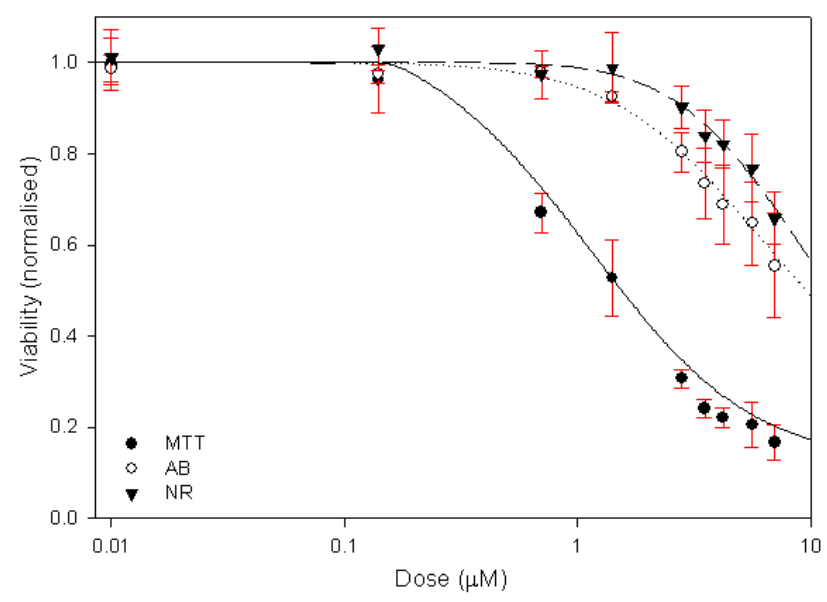

Figure 12-B: $p=3$

C: 


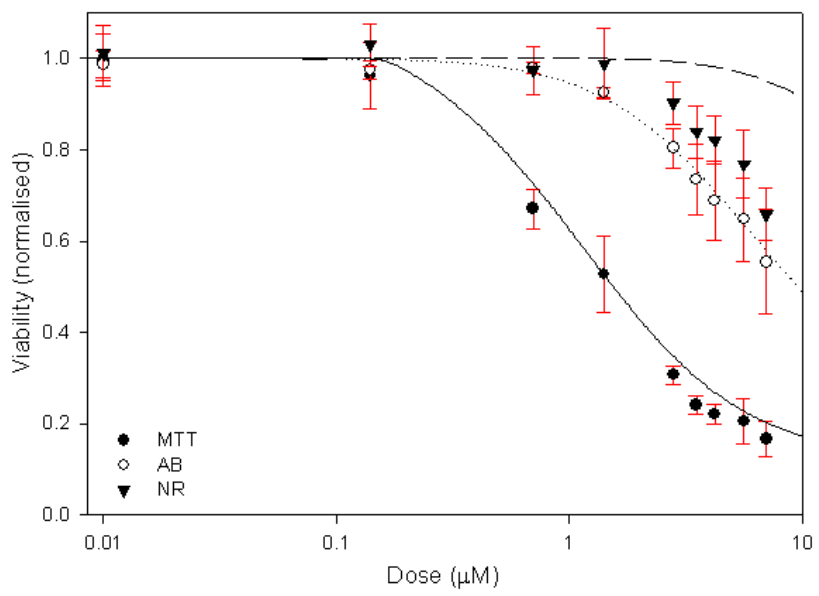

Figure 12-C: $p=4$

Figure S12(A-C): Effect of variation of the parameter $p$ (dashed line) on simulated (lines) viability compared to experimental data (symbols) for 3 different assay types. A: $p=2$, B: $p=3$ and $\mathrm{C}: p=4$. 


\section{References}

SR1. Mukherjee, S.P. and Byrne H.J. Polyamidoamine dendrimer nanoparticle cytotoxicity oxidative stress, caspase activation and inflammatory response: experimental observation and numerical simulation. Nanomed: Nanotech. Bio. Med., 9, 202-211 (2013)

SR2. Salvati, A., et al. Experimental and theoretical comparison of intracellular import of polymeric nanoparticles and small molecules: toward models of uptake kinetics. Nanomed: Nanotech. Bio. Med., 7, 818-826 (2011)

SR3. Mukherjee, S.P., Davoren, M. and Byrne H.J. In vitro mammalian cytotoxicological study of PAMAM dendrimers - towards quantitative structure activity relationships. Toxicol. in Vitro, 24, 169-177 (2010 a)

SR4. Mukherjee, S.P., Lyng, F.M., Garcia, A., Davoren, M. and Byrne H.J. Mechanistic studies of in vitro cytotoxicity of poly(amidoamine) dendrimers in mammalian cells. Toxicol. Appl. Pharm., 248, 259-268 (2010 b)

SR5. Nel, A.E., Mädler, L., Velegol, D., Xia, T., Hoek, E.M.V., Somasundaran, P., Klaessig, F., Castranova, V. and Thompson, M. Understanding biophysicochemical interactions at the nanobio interface. Nature Materials, 8, 543 - 557 (2009)

SR6. Xia, T., Kovochich, M., Brant, J., Hotze, M., Sempf, J., Oberley, T., Sioutas, C., Yeh, J.I., Wiesner, M.R. and Nel, A.E. Comparison of the Abilities of Ambient and Manufactured Nanoparticles to Induce Cellular Toxicity According to an Oxidative Stress Paradigm. Nano Lett., 6, 1794-1807 (2006) 
SR7. Shuvaev, V.V., Han, J., Yu, K.J., Huang, S., Hawkins, B.J., Madesh, M., Nakada, M. and Muzykantov, V.R. PECAM-targeted delivery of SOD inhibits endothelial inflammatory response. FASEB, 25, 348-357 (2011)

SR8. Babior, B.M., Lambeth, J.D. and Nauseef, W. The neutrophil NADPH oxidase. Arch. Biochem Biophys., 397, 342-344 (2002)

SR9. Chamulitrat, W., Stremmel, W., Kawahara, T., Rokutan, K., Fujii, H., Wingler, K., Schmidt, H.H. and Schmidt, R. A constitutive NADPH oxidase-like system containing gp91phox homologs in human keratinocytes. J. Invest. Dermatol., 122, 1000-1009 (2004)

SR10. Bedard, K. and Krause, K.H. The NOX Family of ROS-Generating NADPH Oxidases: Physiology and Pathophysiology. Physiol. Rev., 87, 245-313 (2007)

SR11. Masuko, U.F. Localizing NADPH Oxidase-Derived ROS. Science Signalling: The Signal Transduction Knowledge Environment, 2006(349), re8 (2006)

SR12. Dostert, C., Pétrilli, V., Van Bruggen, R., Steele, C., Mossman, B.T. and Tschopp, J. Innate Immune Activation Through Nalp3 Inflammasome Sensing of Asbestos and Silica. Science, 320, 674-677 (2008)

SR13. Nabeshi, H., Yoshikawa, T., Matsuyama, K., Nakazato, Y., Tochigi, S., Kondoh, S., Hirai, T., Akase, T., Nagano, K., Abe, Y., Yoshioka, Y., Kamada, H., Itoh, N., Tsunoda, S. and Tsutsumi, Y. Amorphous nanosilica induce endocytosis-dependent ROS generation and DNA damage in human keratinocytes. Particle and Fibre Toxicology, 8(1), 10 pages (2011) 